\title{
Conformational rearrangements upon start codon recognition in human 485 translation initiation complex
}

\author{
Sung-Hui Yi ${ }^{1}$, Valentyn Petrychenko ${ }^{2}$, Jan Erik Schliep ${ }^{2}$, Akanksha Goyal ${ }^{1}$, Andreas Linden ${ }^{3,4}$, \\ Ashwin Chari ${ }^{5}$, Henning Urlaub ${ }^{3,4}$, Holger Stark ${ }^{2}$, Marina Rodnina ${ }^{1}$, Sarah Adio ${ }^{6 *}$, Niels \\ Fischer $^{2 *}$
}

${ }^{1}$ Department of Physical Biochemistry, Max Planck Institute for Biophysical Chemistry, Göttingen, Germany

${ }^{2}$ Department of Structural Dynamics, Max Planck Institute for Biophysical Chemistry, Göttingen, Germany

${ }^{3}$ Bioanalytical Mass spectroscopy Group, Max Planck Institute for Biophysical Chemistry, Göttingen, Germany

${ }^{4}$ Bioanalytics, Institute for Clinical Chemistry, University Medical Center Göttingen, Germany

${ }^{5}$ Research Group Structural Biochemistry and Mechanisms, Max Planck Institute for Biophysical Chemistry, Göttingen, Germany

${ }^{6}$ Department of Molecular Structural Biology, Institute for Microbiology and Genetics, Georg-August University of Göttingen, Göttingen, Germany

*Correspondence to: niels.fischer@mpibpc.mpg.de (ORCID 0000-0002-4609-4052) and sarah.adio@uni-goettingen.de (ORCID 0000-0002-6555-0850) 


\begin{abstract}
Selection of the translation start codon is a key step during protein synthesis in human cells. We obtained cryo-EM structures of human $48 \mathrm{~S}$ initiation complexes and characterized the intermediates of codon recognition by kinetic methods using elF1A as a reporter. Both approaches capture two distinct ribosome populations formed on an mRNA with a cognate AUG codon in the presence of elF1, elF1A, elF2-GTP-Met-tRNA ${ }_{i}^{\text {Met, }}$, and elF3. The 'open' $40 \mathrm{~S}$ subunit conformation differs from the human 485 scanning complex and represents an intermediate preceding the codon recognition step. The 'closed' form is similar to reported structures of complexes from yeast and mammals formed upon codon recognition, except for the orientation of elF1A, which is unique in our structure. Kinetic experiments show how various initiation factors mediate the population distribution of open and closed conformations until 60S subunit docking. Our results provide insights into the timing and structure of human translation initiation intermediates and suggest the differences in the mechanisms of start codon selection between mammals and yeast.
\end{abstract}




\section{Introduction}

In eukaryotes, mRNA is recruited to the 435 pre-initiation complex, 43S PIC, which consists of the $40 \mathrm{~S}$ ribosomal subunit, translation initiation factors elF1, elF1A, elF3, elF5, and a ternary complex (TC) composed of eIF2, GTP, and Met-tRNA ${ }_{i}{ }^{\text {Met }}$. 43S PIC binds to the $5^{\prime}$ end of the mRNA and scans along the $5^{\prime}$ untranslated region (5'UTR) in the $3^{\prime}$ direction to find the start codon (AUG) within the context of an optimal Kozak sequence. Start codon recognition stabilizes the $48 \mathrm{~S}$ initiation complex (48S IC), initiates dissociation of elF1, elF1A, elF2, and elF5, and promotes recruitment of the 60S ribosomal subunit to form 80 S IC ready to enter the elongation cycle of protein synthesis. Start codon selection establishes the open reading frame and determines the amino acid sequence of the synthesized protein. The frequency of translation initiation at a given AUG start codon defines translational efficiency of the mRNA, which shapes the composition of the cellular proteome. Compromised fidelity of AUG selection is a common feature of human diseases such as neurodegeneration or cancer (Costa-Mattioli and Walter, 2020; Moon and Parker, 2018; Robichaud et al., 2019).

Translation initiation factors play important roles in mRNA recruitment and start codon selection. elF1A binds to the A site of the 40 S subunit. In yeast, mutations of elF1A and elF1 influence the fidelity of the start codon selection in vivo and in vitro (Fekete et al., 2007; Maag et al., 2006; Nanda et al., 2009; Nanda et al., 2013). Mutations of elF1A N-terminal tail (NTT) enhance leaky scanning, whereby the scanning ribosomes bypass the first AUG start codon and initiate translation at downstream AUG start codons (Fekete et al., 2007). Mutations of elF1A C-terminal tail (CTT) have an opposite (inhibitory) effect on the leaky scanning and enhance initiation at a near-cognate UUG codon within a 5'-proximal Kozak sequence. Mutations of elF1 that change its dissociation rate from the 48S PIC affect the selection of cognate- and near-cognate codons in vitro (Nanda et al., 2013). Premature release of elF1 promotes translation initiation at sub-optimal translation initiation sites (Cheung et al., 2007; Nanda et al., 2009).

In both east and mammals, elF1, elF1A, and (in mammals) elF3 enhance mRNA scanning by inducing an open conformation of the mRNA channel of the $40 \mathrm{~S}$ subunit and by coordinating the TC binding (Brito Querido et al., 2020; des Georges et al., 2015; Hashem et al., 2013a; Hashem et al., 2013b; Kumar et al., 2016; Lomakin and Steitz, 2013; Obayashi et al., 2017; Passmore et al., 2007; Weisser et al., 2013). elF1, which binds to the P site of 40S subunit, interferes with the accommodation of Met-tRNA ${ }_{i}{ }^{M e t}$ in the $P$ site during scanning. 
Upon codon recognition, elF1 is released from the ribosome. In mammals, elF1 and eIF1A act synergistically to mediate the assembly of h48S IC on the initiation codon and enhance binding affinities of each other to the 40S subunit (Pestova et al., 1998; Sokabe and Fraser, 2014). elF1 inhibits premature GTP hydrolysis by elF2 until an AUG codon is recognized (Brito Querido et al., 2020; Lomakin and Steitz, 2013; Unbehaun et al., 2004). In the absence of elF1 or eIF3, h48S IC does not scan mRNA and remains in the proximity of the mRNA $5^{\prime}$-end (Kumar et al., 2016). In the late stage of h48S IC formation, elF1A competes with elF5 for binding to elF5B, allowing dissociation of elF5-elF2 complex after GTP hydrolysis by elF2 (Lin et al., 2018).

elF5 is a GTPase-activating protein (GAP) of elF2. In yeast, elF5 binds to elF2 during scanning and induces GTP hydrolysis by elF2, but the reaction product, inorganic phosphate (Pi) remains bound until the ribosome recognizes the start codon, which triggers Pi release and the subsequent elF2 dissociation (Algire et al., 2005; Algire et al., 2002; Llacer et al., 2018). By displacing elF1, elF5 facilitates the accommodation of Met-tRNA ${ }_{i}{ }^{\text {Met }}$ and enables gated release of Pi from elF2, which effectively ends the scanning process (Algire et al., 2005; Llacer et al., 2018; Luna et al., 2013; Luna et al., 2012; Saini et al., 2014). This is different in mammals, where GTP hydrolysis by elF2 is induced upon codon recognition by relieving inhibition by elF1 (Pestova and Kolupaeva, 2002; Pisareva and Pisarev, 2014; Unbehaun et al., 2004). Finally, elF5B is a translational GTPase that facilitates 60S subunit docking (Acker et al., 2009; Pestova et al., 2000; Pisareva et al., 2007; Wang et al., 2019). Recruitment of 60S subunit induces dissociation of the remaining initiation factors and marks the onset of translation elongation.

Structural and biochemical work suggests that the $48 \mathrm{~S}$ complexes adopt different conformations during scanning and upon start codon recognition. The structure of yeast $48 \mathrm{~S}$ IC trapped on a near-cognate AUC instead of the AUG codon in the mRNA ( $y 48 S$ AUC) shows an 'open' 40S conformation with Met-tRNA ${ }_{i}{ }^{M e t}$ in the $P_{\text {out }}$ State (Hussain et al., 2014; Llacer et al., 2015). In this intermediate, scanning is stopped by partial base pairing of Met-tRNA ${ }_{i}{ }^{M e t}$ with the AUC codon. AUG recognition induces the accommodation of Met-tRNA ${ }_{i}{ }^{\text {Met }}$ into the $P$ site $\left(P_{\text {in }}\right.$ state) and tightens the mRNA binding channel around the anticodon stem, forming a 'closed' state of the y48S AUG (Hussain et al., 2014; Llacer et al., 2015; Llacer et al., 2018). This conformation is stabilized by contacts between the NTT of elF1A with the tRNA-mRNA duplex (Hussain et al., 2014; Llacer et al., 2015; Llacer et al., 2018). AUG recognition induces the release of elF1 and elF2 $\beta$ from the P site, Pi release from elF2, and reorganization of elF3 
(Algire et al., 2005; Kapp and Lorsch, 2004; Llacer et al., 2015; Llacer et al., 2018; Maag et al., 2005). The existing structures of mammalian 48 S IC assembled on AUG are similar to the closed y48S AUG (Eliseev et al., 2018; Simonetti et al., 2020). Recent structural work has captured human 48S complex in the course of mRNA scanning (Brito Querido et al., 2020), which shows a distinct 405 subunit conformation and no codon-anticodon recognition in the $\mathrm{P}$ site. One important difference between yeast and mammalian 48S IC assembly concerns the role of eIF3 in AUG recognition. Yeast elF3 appears to be not essential (Maag et al., 2006), whereas mammalian elF3 is indispensable for h43S PIC and h48S IC formation (Kumar et al., 2016; Pestova and Kolupaeva, 2002; Sokabe and Fraser, 2014; Sokabe et al., 2012). While high-resolution structures and detailed biochemical studies of y48S IC provide important insights into the mechanism of start codon selection in yeast, structural view on the process in higher eukaryotes is just starting to emerge. The kinetic data on translation initiation in mammals are scarce compared to the detailed analysis available for the yeast system (Algire et al., 2005; Kapp and Lorsch, 2004; Llacer et al., 2015; Llacer et al., 2018; Maag et al., 2005), which calls for time-resolved studies in the mammalian system.

Here we use a fully reconstituted in vitro translation system from human cells to study the assembly of human 48 S IC by single-particle cryo-EM and rapid kinetic approaches. Our cryoEM data show two distinct conformations of the human $48 \mathrm{~S}$ complex assembled on an mRNA with a cognate AUG start codon, prior to and after codon-anticodon recognition. Although both complexes contain the same set of initiation factors, e.g. eIF1A, eIF1, TC, and eIF3, their arrangement at the decoding center and the conformations of the $40 \mathrm{~S}$ subunit are different. Comparison to previously reported structures, in particular the open and closed y48S, as well as h48S scanning complexes, suggests that the open h48S AUG may represent an early intermediate of the codon recognition process. Kinetic analysis using elF1A dissociation as a diagnostic assay for changes in ribosome conformations suggests how elFs and start codon recognition remodel the ribosome from 43 S PIC and 48S PIC to 48S IC and finally, how elF5B remodels 48 S IC conformation for the 60 S subunit joining. This work provides further evidence for the conserved and distinct features of start codon-recognition mechanism in mammals and yeast. 


\section{Results}

\section{Structures of h48S AUG in the open and closed state}

To analyze 48S IC assembled on the AUG start codon by cryo-EM, we reconstituted complexes using purified human 40 S subunits, elF1, elF1A, elF2-GTP-Met-tRNA ${ }_{i}{ }^{\text {Met }}$ (TC), elF3, in the presence of elF4A and $4 \mathrm{~B}$, and in-vitro transcribed mRNA (Figure 1 - figure supplement 1 and Methods) (Pestova et al., 1998; Pestova and Kolupaeva, 2002; Pisarev et al., 2007). The model mRNA (Figure 1 - figure supplement 2A) has an unstructured 5'UTR, which overcomes the requirement for 5 ' capping and elF4F recognition, followed by an optimal Kozak sequence, a short open reading frame coding for a peptide MVRFKA, and a 3'UTR sequence that serves as a primer binding site for the primer-extension inhibition (toe-printing) assay (Pestova and Kolupaeva, 2002). Functional activity of $48 \mathrm{~S}$ and $80 \mathrm{~S}$ complexes was verified by toe-printing (Figure 1 - figure supplement 2A). In mammals, elF1, elF1A, TC and elF3 are the minimal set of essential factors to reconstitute the $48 \mathrm{~S}$ IC on an unstructured and uncapped mRNA (Pestova et al., 1998; Pestova and Kolupaeva, 2002) (Figure 1 - figure supplement 2B). 48S IC causes a strong toe-printing stop on the cognate start codon (AUG), which is not observed with the non-cognate codon (CUC), while on the near-cognate codon (AUC) $48 \mathrm{~S} \mathrm{IC} \mathrm{forms} \mathrm{a}$ specific, but labile complex (Figure 1 - figure supplement $2 \mathrm{C}$ ). These human $48 \mathrm{~S}$ initiation complexes formed in the presence of eIF1A, eIF1, TC, elF3 but in the absence of elF5 or elF5B are denoted in the following as h48S AUG, h48S CUC and h48S AUC.

For the cryo-EM work on h48S AUG complexes, we used the GraFix procedure (Kastner et al., 2008) to stabilize the complexes by crosslinking with bis(sulfosuccinimidyl)suberate (BS3), p-maleimidophenyl isocyanate and glutaraldehyde. After sorting cryo-EM images according to the $40 \mathrm{~S}$ subunit conformation and the presence of elFs (Figure 1 - figure supplement 3 and Methods), we obtained two final cryo-EM reconstructions depicting h48S AUG in an open and closed state, respectively (Figure 1 and Supplementary table 1). In both states, we were able to model almost all components of the complex, except for elF1 and the C-terminal domain of elF $2 \beta$ in the closed state (see below). In the final structures, the occupancy of 405 subunits with elF3 was high $\geq 80 \%$ (estimated by in silico sorting of cryo-EM data, see Methods for details) and almost all 13 subunits of elF3 could be placed, except for the flexible peripheral subunit elF3i (Brito Querido et al., 2020) and the loosely associated subunit elF3j (Fraser et al., 2007; Sokabe and Fraser, 2017). Analysis of the h48S AUG complexes using crosslinking mass spectrometry (XLMS) supported our structural models (Figure 1 - figure supplement 4, 
Supplementary tables 2-5 and Methods). 91\% of the crosslinks fall into the $30 \AA$ range for both the crosslinkers used (BS3 and LC-SDA). Crosslinks that exceed the distance restrictions (35-45 ̊̊) can be explained by local flexibility and dynamic regions of the structures. We note that more crosslinks could be mapped onto the open state, especially because elF1 and some parts of elF3c could only be modeled in the open state (see below).

A

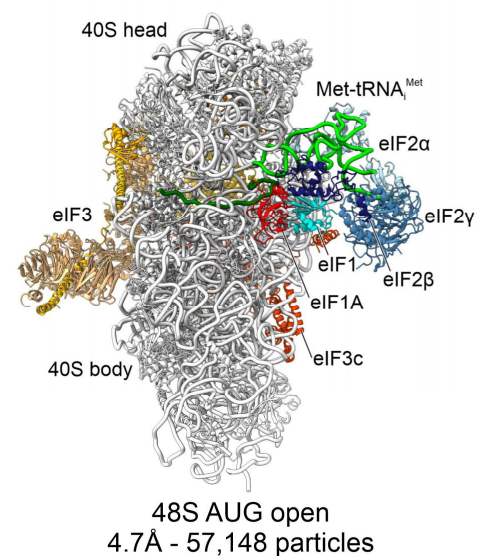

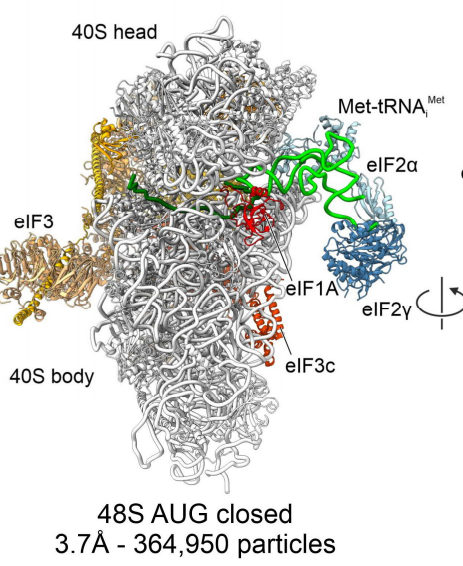

$3.7 \AA-364,950$ particles

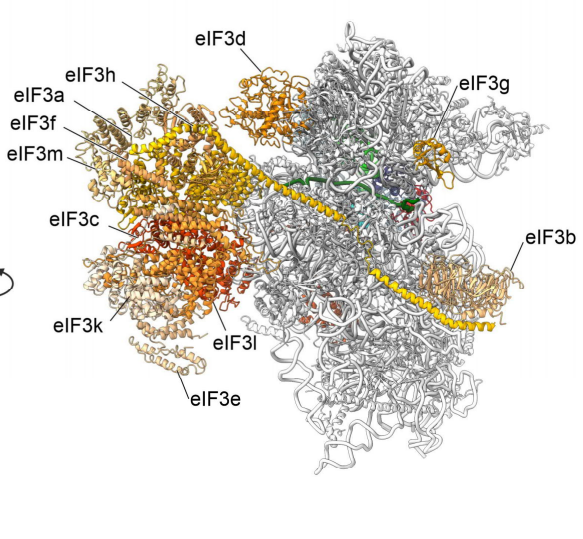

Figure 1. Structures of the h48S AUG complex in open and closed states.

(A) Overall structures of the open (left) and closed (right) states assembled on mRNA with cognate AUG start codon. Structures are shown as ribbon models with the respective resolution and particle numbers used for the final cryo-EM maps. In the closed state, the density for elF1 and elF2 $\beta$ is missing. (B) Structural assignment of elF3 subunits in h48S AUG closed. All subunits of elF3 could be placed except for elF3i and eIF3j. Individual subunits of elF3 are color-coded as indicated. 
A

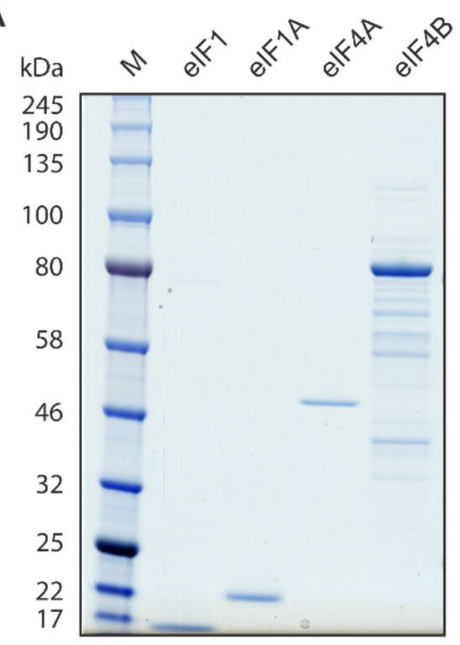

C

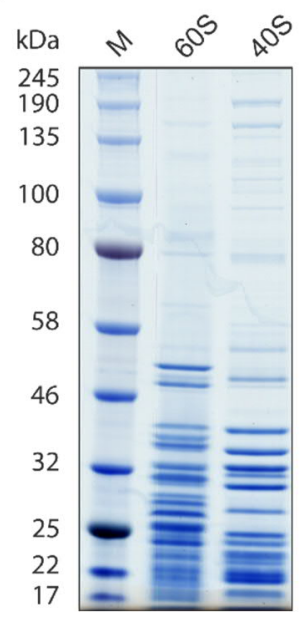

B

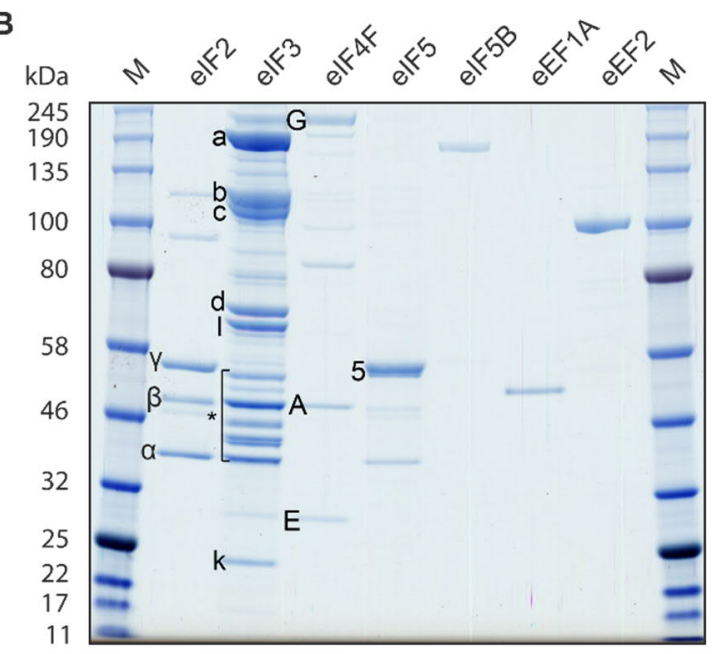

D

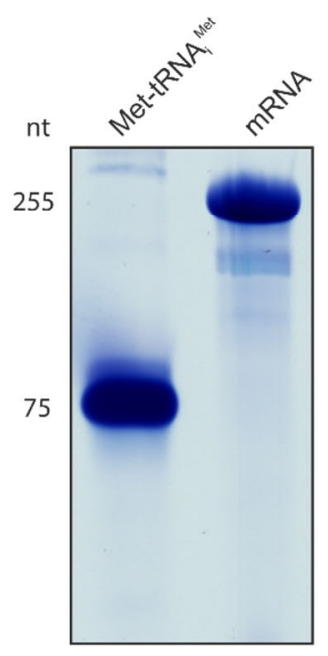

Figure 1 - figure supplement 1. Components for the reconstituted in vitro translation system.

(A) Recombinant human IFs: elF1 (molecular weight $12.7 \mathrm{kDa}$ ), elF1A (16.5 kDa), elF4A (46.2 kDa) and elF4B (69.2 kDa) expressed in E. coli.

(B) Native initiation and elongation factors from mammalian cells. Proteins prepared from HeLa cells: elF2 comprising $2 \alpha$ (36 kDa), $2 \beta$ (38 kDa), and $2 \gamma$ (52 kDa) subunits; elF3 subunits 3a (167 kDa), 3b (92 kDa), 3c (105 kDa), 3d (64 kDa), 3e (52 kDa), 3f (38 kDa), 3g (36 kDa), 3h (40 kDa), 3i (36 kDa), 3j (29 kDa), 3k (25 kDa), 3l (67 kDa), 3m (43 kDa), *elF3 subunits 3e to 3j are indicated in the bracket; elF4F subunits 4G (175 kDa), 4A (46 kDa), 4E (25 kDa); elF5B (138.8 kDa), and elongation factors, eEF1A (50 $\mathrm{kDa})$ and eEF2 (95 kDa); elF4F was not used in this work. Native elF5 (49.2 kDa) was purified from rabbit reticulocyte lysate. (C) Proteins of 605 and $40 S$ ribosomal subunits from HeLa cells. (D) In vitro transcribed human initiator $\operatorname{tRNA} A_{i}^{\text {Met }}$ and model mRNA. The tRNA ${ }_{i}^{\text {Met }}$ was aminoacylated with $\left[{ }^{3} \mathrm{H}\right]-$ methionine and purified. 
A

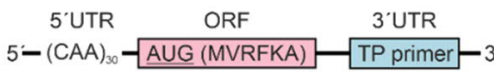

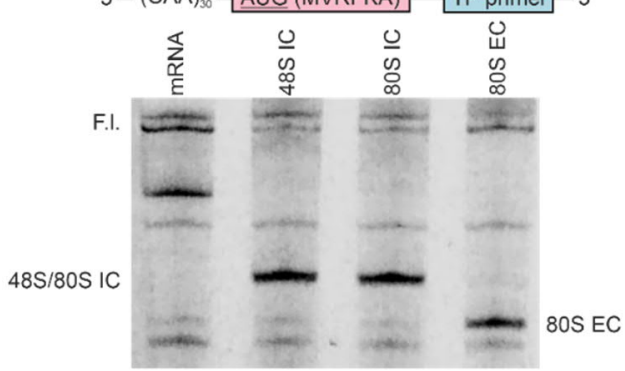

B

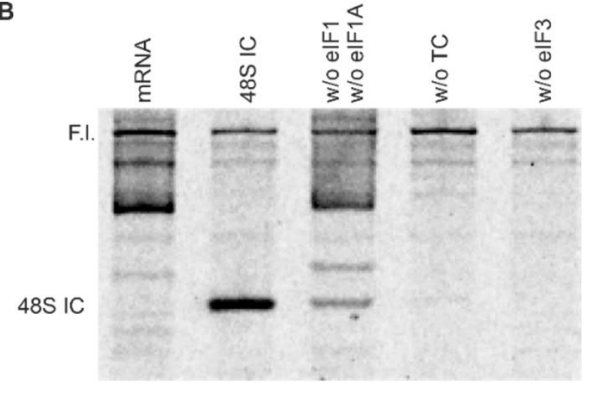

C

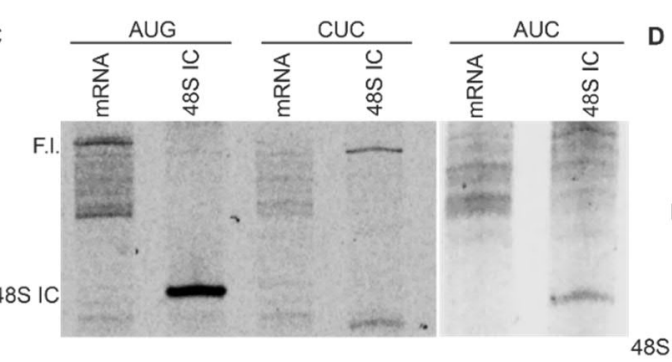

D

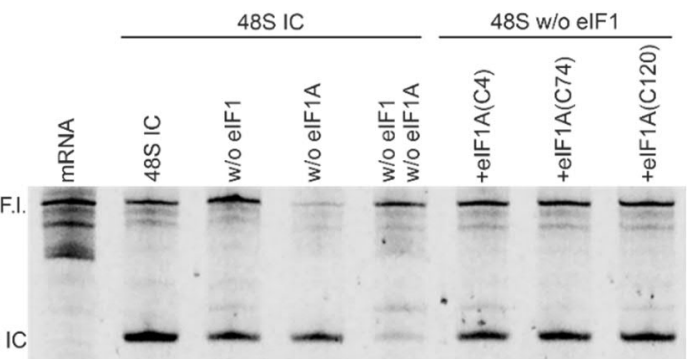

Figure 1 - figure supplement 2. Fluorescence toe-printing assays of reconstituted h48S IC, h80S IC and h80S EC.

(A) Schematic of the model mRNA and the toe-printing assay of the model mRNA without ribosome complex, and with h48S IC, h80S IC and h80S EC. The model mRNA is uncapped and has an unstructured 5'UTR containing 30 CAA repeats followed by an ORF coding for a short peptide MVRFKA and a toe-printing (TP) primer binding site in the 3'UTR. Toe-printing primer was labeled at the 5' end by Atto647. The h48S IC was assembled from 40S subunits, elF1, elF1A, TC, elF3, elF4A and elF4B in reaction buffer containing GTP. The h80S IC is assembled by adding elF5, elF5B and 60S subunits to h48S IC. 80 S EC was formed upon synthesis of the MVRFKA peptide after addition of eEF1A, eEF2 with respective aminoacyl-tRNAs. F.I. is the full-length cDNA product of RT-reaction with the model mRNA as template.

(B) Toe-printing assay of h48S IC in the absence (w/o) of individual elFs.

(C) Toe-printing of h48S IC assembled on AUG, CUC and AUC mRNA. No toe-print was detected for $48 \mathrm{~S}$ CUC PIC and a weak CDNA band was found for 48S AUC PIC.

(D) Toe-printing assay of h48S IC formed with elF1A derivatives. The WT elF1A was replaced by eIF1A derivatives with a cysteine at position 4, 74, or 120 . Because the presence of either elF1 or elF1A alone can promote h48S IC formation on the model mRNA, the activity of elF1A derivatives was tested in the absence of elF1. 
A

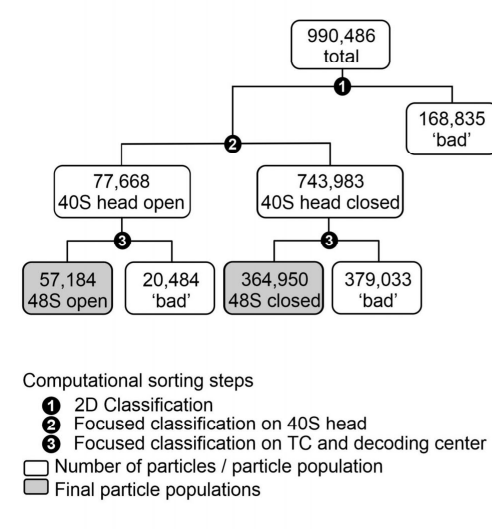

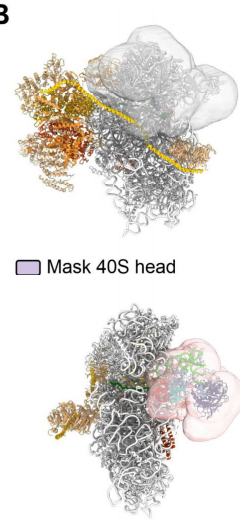

$\square$ Mask TC and DC
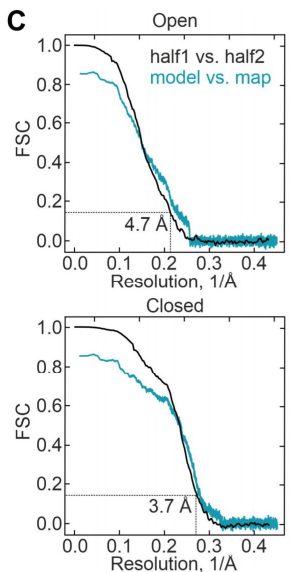

D

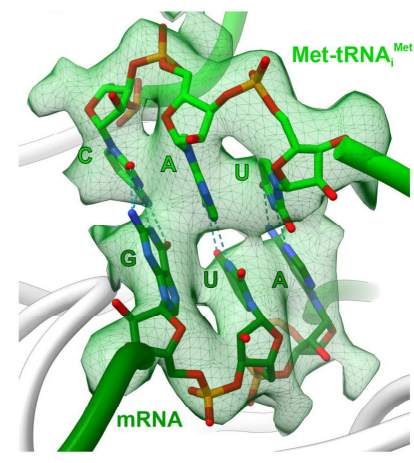

E
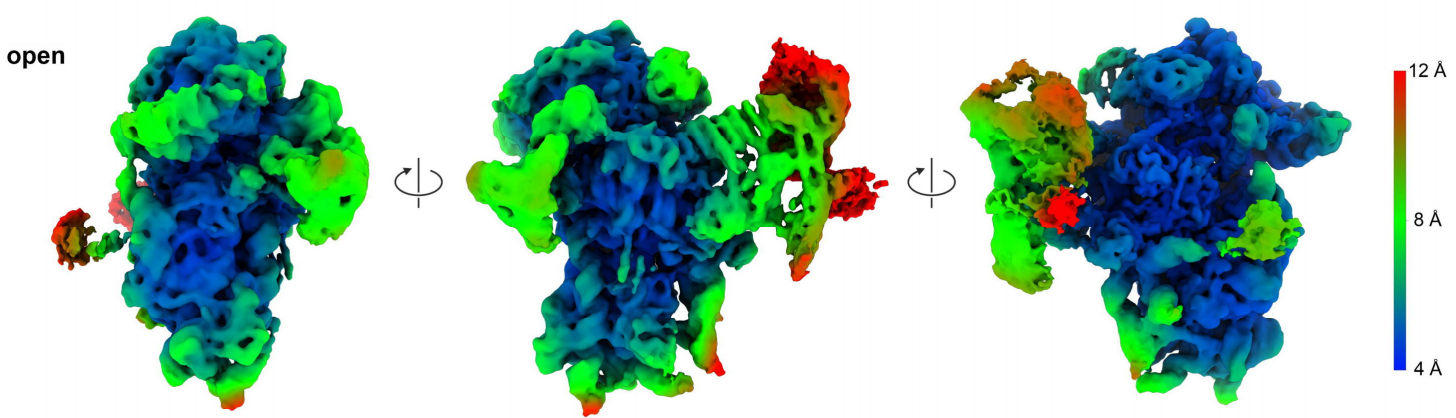

closed
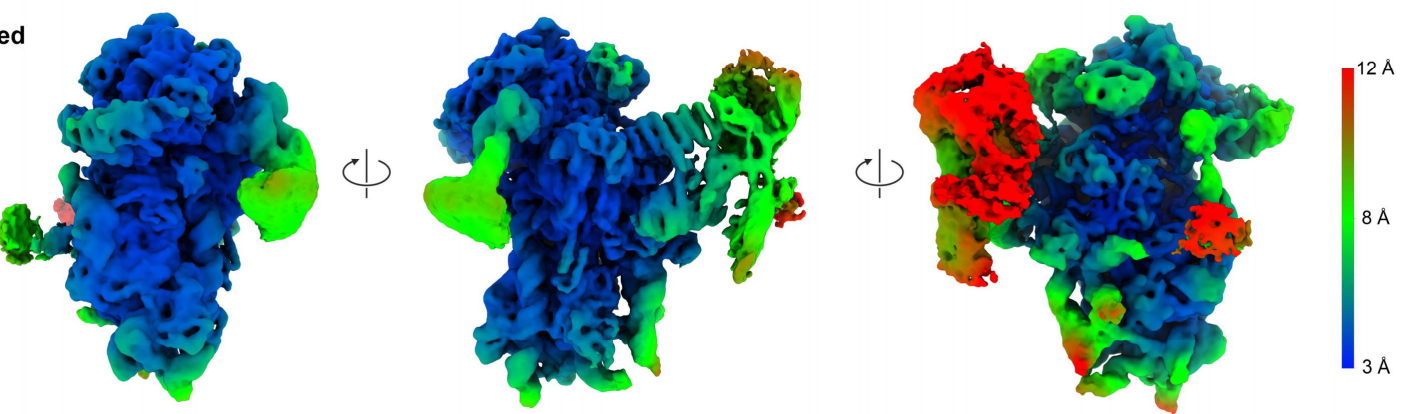

\section{Figure 1 - figure supplement 3. Cryo-EM data processing.}

(A) Sorting scheme for global and focused classifications of cryo-EM data.

(B) Masks used for particle signal subtraction and focused classifications on 40S head (top) and ternary complex together with decoding center (bottom); DC, decoding center.

(C) Fourier-Shell-Correlation (FSC) curves of the final cryo-EM reconstructions.

(D) Details of the codon-anticodon interaction indicating start codon recognition in the closed state. The cryo-EM map (green transparent surface) is shown at $3 \sigma$.

(E) Unsharpened cryo-EM maps of the open (top) and closed state (bottom) colored according to local resolution. Left: View onto TC and decoding center; center: View onto solvent site; right: View onto elF3 core. Note the substantially lower local resolutions of elF2 $\gamma$ and elF3; atomic models for such regions were refined at lower resolution (see Methods). 

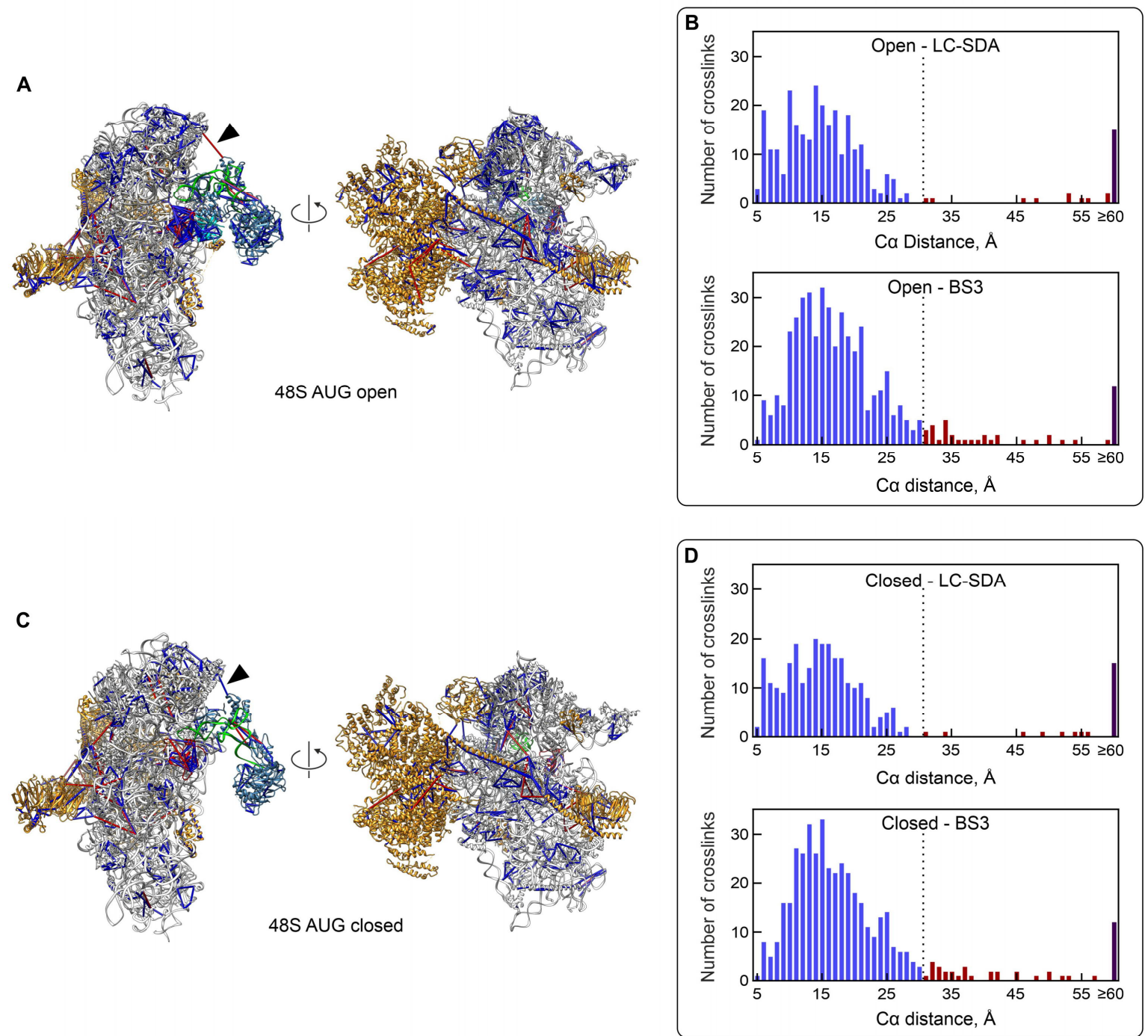

Figure 1 - figure supplement 4. Crosslinking-mass spectrometry analysis.

(A) and (C) Crosslinks mapped on the models of the open (A) and closed state (C), shown in two different orientations. Crosslinked residues are indicated by rods, colored in blue for permitted distances (<30 $\AA$ ) and in red for non-permitted distances (30-45 $\AA$ ); crosslinks for distances larger than $45 \AA$ are not shown. The $40 \mathrm{~S}$ subunit is depicted in white, Met-tRNA ${ }_{i}{ }^{\text {Met }}$ in green, elF1 in cyan, elF1A in red, elF2 in steel blue and elF3 in orange. The black arrow head marks the change in the crosslink distance between elF2 $\alpha$ (Lys123) and ribosomal protein S25 (Lys57) upon head closure.

(B) and (D) Histograms of distances for unique crosslinks mapped onto the open (B) and closed states (D) obtained with the crosslinkers LC-SDA (top) and BS3 (bottom), respectively. Crosslinking distances longer than $45 \AA$ account for approx. $6 \%$ of the crosslinks and most likely result from transient interactions of proteins not bound to the 40S subunit. 
The overall conformation of the $40 \mathrm{~S}$ subunit is clearly different in the open and closed states (Figure 2A and Figure 2 - figure supplement 1). The $4.7 \AA$ cryo-EM structure of the h48S AUG open represents a minor population of the complexes ( 16\%, Figure 1$)$ that do not appear to form the codon-anticodon interactions Figure 2B), although the mRNA contains the AUG codon and all the initiation components are present (Figure 1). In our open state, the 405 head domain is tilted towards the solvent site, which opens the decoding center (Figure $2 \mathrm{~A}$ and Figure 2 - figure supplement $1 \mathrm{~A}$ ) and the mRNA entry latch (Figure $2 \mathrm{C}$ ). Probably due to the absence of codon-anticodon interaction, only the mRNA backbone is resolved in $\mathrm{h} 48 \mathrm{~S}$ AUG open; the lack of information for the bases prevents identification of the codon in the $P$ site. Met-tRNA ${ }_{i}^{\text {Met }}$ is in the $\mathrm{P}_{\text {out }}$ conformation and contacts the $40 \mathrm{~S}$ head domain. Specifically, two conserved GC base pairs in the tRNA anticodon stem interact with G1639 to A1641 in helix 29 (h29) of 18S rRNA mostly via unspecific RNA-RNA backbone interactions (Figure 2C,D), similarly to the interactions observed in yeast (Llacer et al., 2015). elF2 $\alpha$ and elF2 $\beta$ reach towards the decoding center and interact with the tRNA, whereas elF2 $\gamma$ points away from the 40 S subunit body (Figure 2C). elF1 binds close to the P-site codon, while the C-terminal domain (CTD) of elF2 $\beta$ interacts with elF1 and contacts the anticodon-stem loop of the tRNA, thereby stabilizing the tRNA $P_{\text {out }}$ conformation (left panels in Figure 2B,D). elF1A binds to the A site of the decoding center between h18 and h44 of 18S rRNA and proteins uS12 and eS30 of the $40 \mathrm{~S}$ body domain (Figure 2E,F and Figure 2 - figure supplement $1 \mathrm{~B}$ ), as in all reported 48S IC structures (Brito Querido et al., 2020; Llacer et al., 2015; Llacer et al., 2018; Simonetti et al., 2020). An $\alpha$-helical element (residues 265-278) in the N-terminal domain (NTD) of elF3c, which is specific for human elF3, interacts with elF1 in a similar way as in the h48S.scan complex (Figure 2C and Figure 2 - figure supplement 1C; (Brito Querido et al., 2020)). 
A

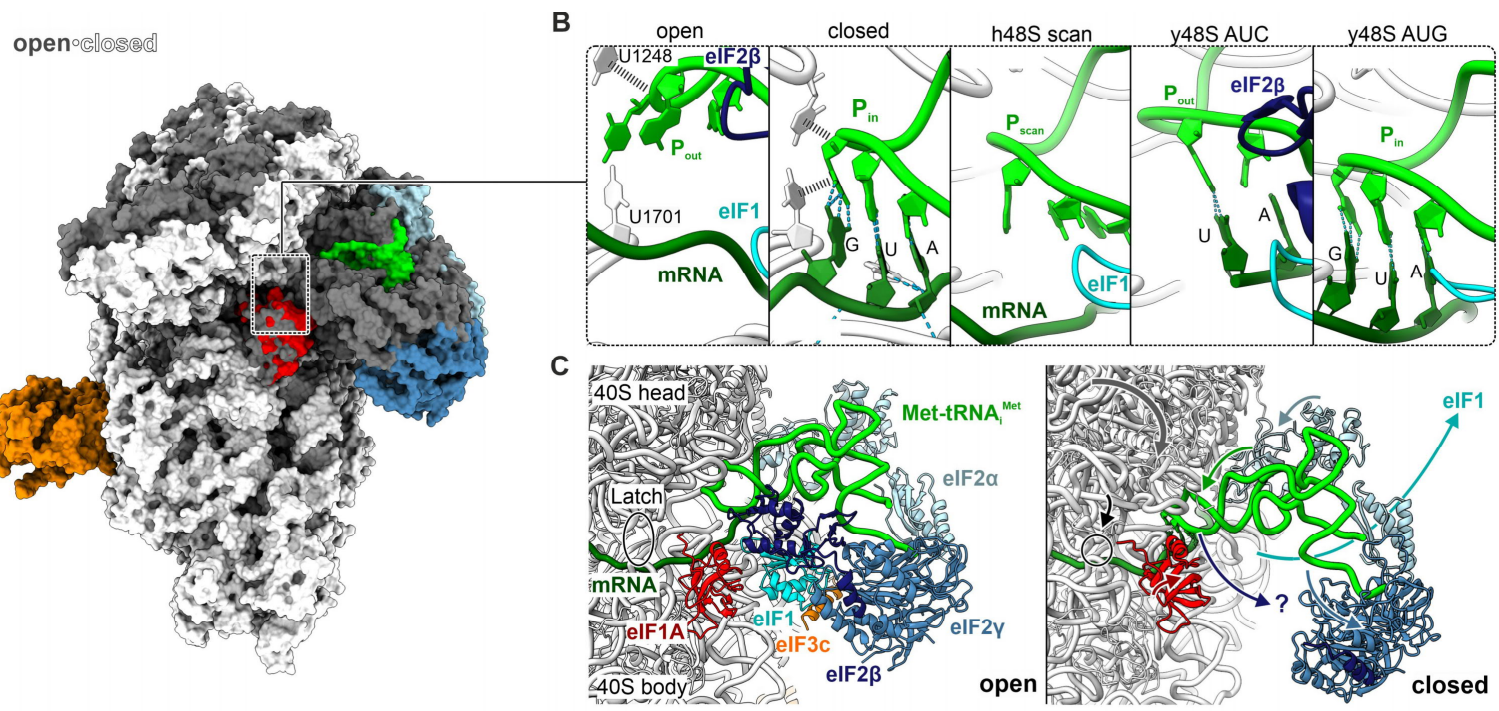

D

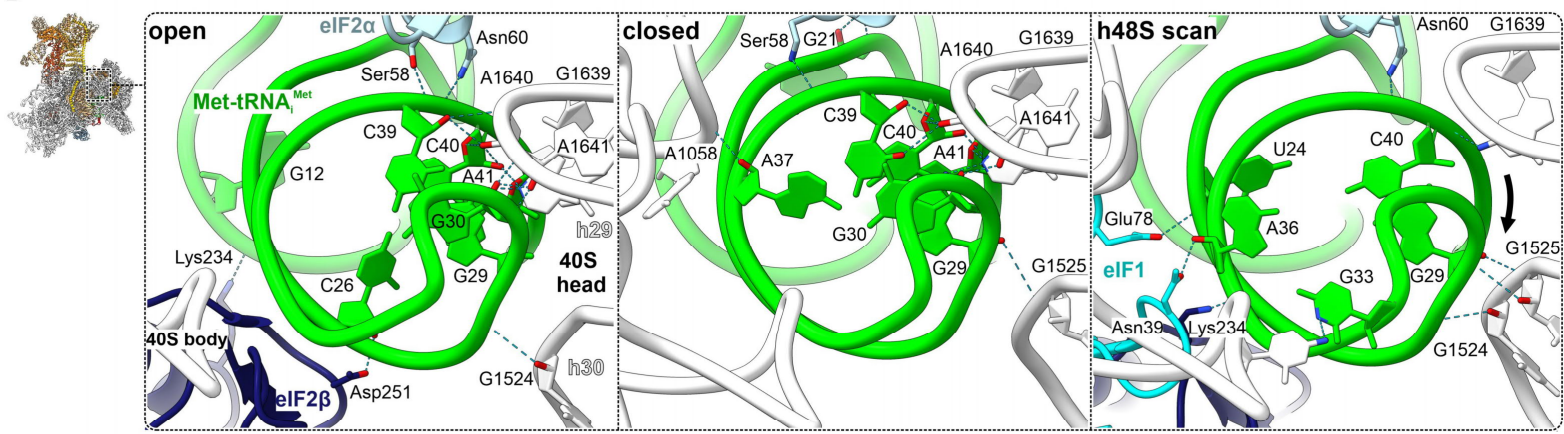

E

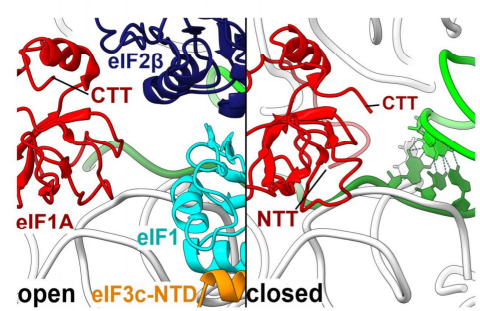

F open
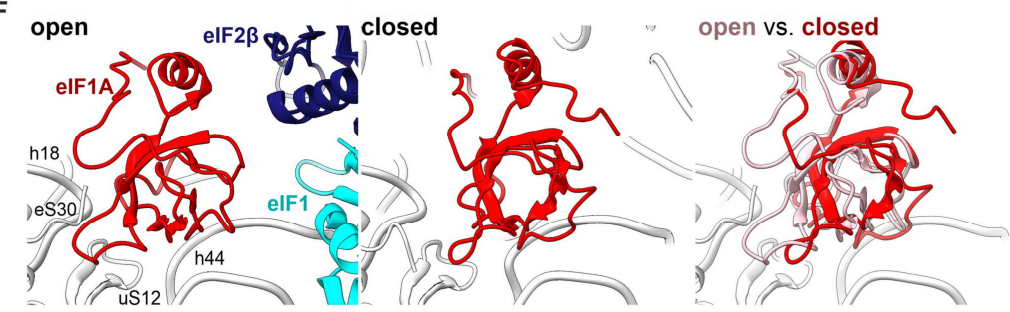

Figure 2. Two distinct conformations of the h48S AUG complex.

(A) Superposition of the h48AUG in the open state (all components of the complex are shown in grey) and the closed state (40S subunit, white; elFs colored as in Fig. 1: elF1A, red; Met-tRNA ${ }_{i}^{\text {Met }}$, green; elF2 $\alpha$, light blue; elF2 $\gamma$, blue; elF3, orange); atomic models for both complexes are shown in space fill representation. Here and in other Figures, structures were aligned by the $40 \mathrm{~S}$ body domain, if not mentioned otherwise.

(B) Close-ups of the P site in 48S IC complexes from human and yeast. Open, closed: h48S AUG states described here. h48S scan: human 48S IC with non-cognate codon CUC replacing the AUG codon (PDB 6ZMW, (Brito Querido et al., 2020)). y48S·AUC: yeast 48S IC open state with a near-cognate AUC codon (PDB 3JAP, (Llacer et al., 2015)). y48S·AUG: yeast 48S IC with cognate AUG and elF1 bound in closed state (PDB 3JAQ, (Llacer et al., 2015)). Note the repositioning of both Met-tRNA ${ }_{i}{ }^{\text {Met }}$ from $P_{\text {out }} / P_{\text {scan }}$ to $P_{\text {in }}$ and mRNA upon 40S head domain closure resulting in codon-anticodon interaction. 
(C) Rearrangements of the TC in the open vs. closed h48S AUG. Arrows mark major conformational changes upon $40 \mathrm{~S}$ head (grey) movement from open to closed state: closure of the mRNA entry latch (black circle); movement of Met-tRNA ${ }_{i}^{\text {Met }}$ (green) and elF2 $\alpha$ (light blue) towards and of elF2 $\gamma$ away from the 40 S subunit; dissociation of elF1 (cyan) and movement of elF2 $\beta$ (purple) away from the $P$ site; and repositioning of elF1A (red).

(D) Interactions between the ASL of Met-tRNA ${ }^{\text {Met }}$ (green) with the $40 \mathrm{~S}$ head domain (18S rRNA, white). Note that tRNA contacts in the h48S AUG open and closed states are similar, but different in h48S scan state (PDB 6ZMW (Brito Querido et al., 2020)). Dashes indicate residues that are within H-bond distance. Arrow marks the shift in interactions from h29 to h30. Structures were aligned by the ASL of initiator tRNA.

(E) Close-ups of the decoding center. Upon 40S head closure, eIF1 and eIF2 move out of the decoding center, while elF1A and its N-terminal tail (NTT) move towards the P site. Note the $\alpha$-helix in the NTD of elF3c (elF3c-NTD), which interacts with elF1 in the open state and is not resolved in the closed state. (F) Repositioning of elF1A. Position and interactions of elF1A in the open (left) and closed states (center), and superposition (right) of elF1A in open (light red) and closed state (red). uS12 and eS30, proteins of the 40 S subunit; h44, helix 44 of 18 S rRNA. 
A

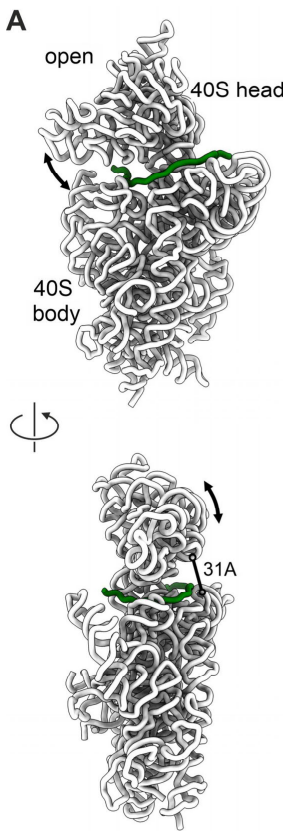

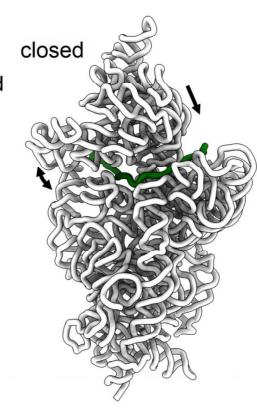

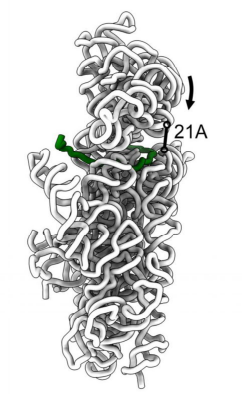

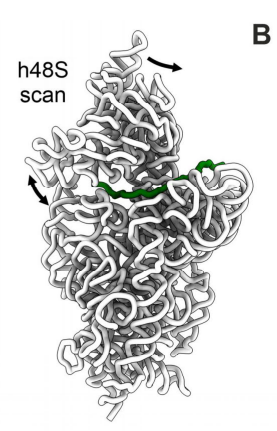

B

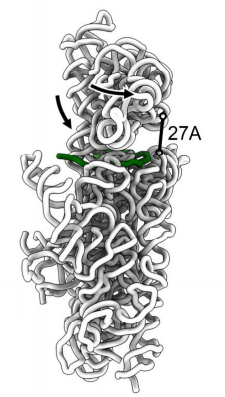

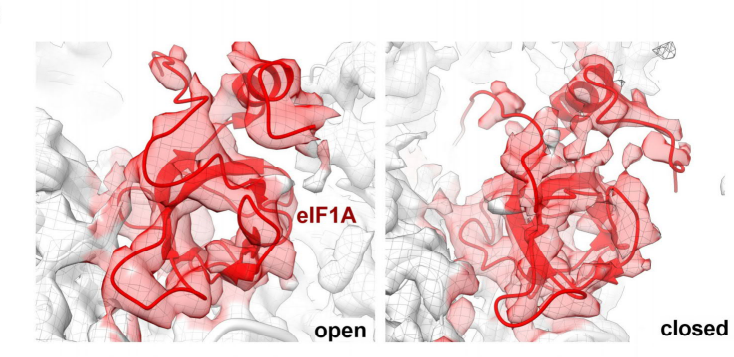

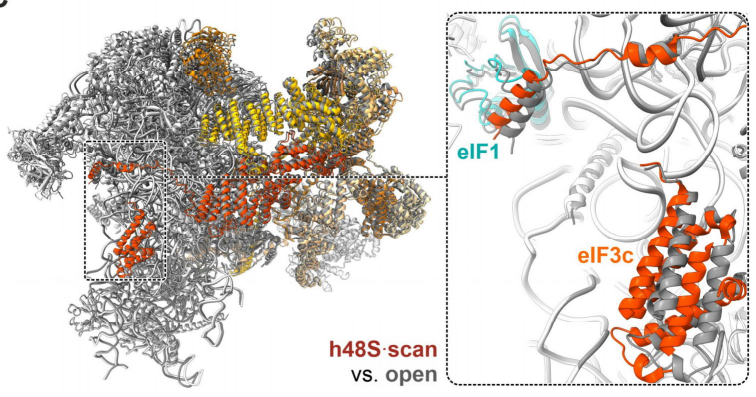

Figure 2 - figure supplement 1. Structural rearrangements in h48S AUG IC.

(A) Conformation of the $40 \mathrm{~S}$ subunit in open, closed and scanning states. h48S scan, PDB 6ZMW (Brito Querido et al., 2020). For visual clarity, only the $18 \mathrm{~S}$ rRNA (white) is shown with the mRNA (green). Arrows denote major modes of motion. Distances are measured between residues A1058 and A1640 of $18 \mathrm{~S}$ rRNA.

(B) Cryo-EM map of eIF1A in the open vs. closed state. The experimental map (transparent) is shown at $3 \sigma$ with the ribbon models of elF1A (red) and $18 \mathrm{~S}$ rRNA (white).

(C) elF3 conformation. Left: Superposition of open h48S AUG (light grey) with h48S scan (white, elF3 subunits in colors, PDB 6ZMW (Brito Querido et al., 2020)) revealing a similar overall elF3 structure. Right: close-up showing a distinct orientation of the $\alpha$-helix of elF3c, which moves with elF1, and a slight shift of its NTD between the two states.

The major population of h48S AUG ( 84\%) adopts a closed state, denoted as h48S AUG closed. The structure, obtained at $3.7 \AA$ A resolution, shows the closure of the $40 \mathrm{~S}$ head domain and of the mRNA entry latch upon start codon recognition (Figure 2A-D and Figure 2 - figure supplement $1 \mathrm{~A}$. Met-tRNA ${ }_{i}{ }^{\text {Met }}$ moves into the $P$ site $\left(P_{i n}\right)$ and the mRNA changes its path to base pair with the tRNA (Figure $2 \mathrm{~B}$ ). The TC, and in particular $\mathrm{TRNA}_{i}{ }^{\mathrm{Met}}$, does not change its interactions with the $40 S$ head domain, but moves together with it upon 405 subunit closure (Figure 2D). elF1 and the CTD of elF2 $\beta$ appear to move out of the decoding center, as the respective density is not traceable in the closed state (Figure $2 \mathrm{C}$ ). Upon release of elF2 $\beta$ from the decoding center, the peripheral part of the TC, in particular elF2 $\alpha$ and the acceptor stem 
of Met-tRNA ${ }_{i}{ }^{\text {Met }}$, become more flexible and are not as well defined as in the open state. In the closed state there is also no density for the $\alpha$-helical element of elF3c, which appears to dissociate together with elF1 from the decoding center upon start codon recognition. Moreover, formation of the closed state induces a rearrangement of elF1A, which now lacks the contacts with h18 and eS30 and moves closer to the P site where its $\mathrm{N}$-terminal tail reaches towards the tRNA (Figure 2E,F and Figure 2 - figure supplement $1 \mathrm{~B}$ ).

\section{Comparison to reported 48 S IC structures}

The h48S AUG open state, which was not captured in other 48S IC structures with AUG, is similar to the near-cognate y48S AUC complex (Llacer et al., 2015)(Figure 3A). The main difference is in mRNA-tRNA interactions, as in the y48S AUC complex the anticodon of tRNA $\mathrm{A}_{\mathrm{i}}$ Met interacts with the $\mathrm{U}$ of the near-cognate $\mathrm{AUC}$ codon (Figure $2 \mathrm{~B}$ ), whereas in $\mathrm{h} 48 \mathrm{~S}$ AUG open complex the mRNA takes a different path and appears too far away from $\operatorname{tRNA}_{i}{ }^{\text {Met }}$ for base pairing. The present h48S AUG closed state resembles previous high-resolution structures of 48 S IC obtained with a cognate AUG codon, both from yeast (y48S AUG, (Llacer et al., 2015; Llacer et al., 2018)) and rabbit (r48S AUG, assembled on $\beta$-globin mRNA, (Simonetti et al., 2020)), except for the orientation of elF1A (see below) (Figure 3A and Figure 3 - figure supplement $1 A)$. Notably, closed y48S AUG contains elF5, which replaces elF1 and appears to stabilize elF2 $\beta$ and elF2 $\alpha$ (Llacer et al., 2018) similarly to elF1 in the h48S AUG open state. In y48S AUG, two slightly distinct positions of elF2 $\gamma$ were identified that differ from the present h48S AUG closed state by reaching further towards the decoding center, most likely due to the interactions of elF5 with the decoding center in the yeast complexes (Llacer et al., 2015; Llacer et al., 2018). Comparing r48S AUG with the h48S AUG closed complex, the similarities are in the overall $40 \mathrm{~S}$ subunit conformation and the positions of Met-tRNA ${ }_{i}{ }^{\text {Met }}$. In both structures the density for elF1, elF2 $\beta$, elF3i and elF3j are not resolved. However, there are also differences, for example, a somewhat different orientation of elF2y, elF2 $\alpha$ and elF3, as well as the absence of density for elF3b, NTD of elF3c and CTD of elF3h in the r48S AUG structure (Simonetti et al., 2020). We note that in contrast to our fully reconstituted h48S complexes, r48S AUG contains ABCE1, a ribosome recycling factor (Pisarev et al., 2010; Schuller and Green, 2017). 

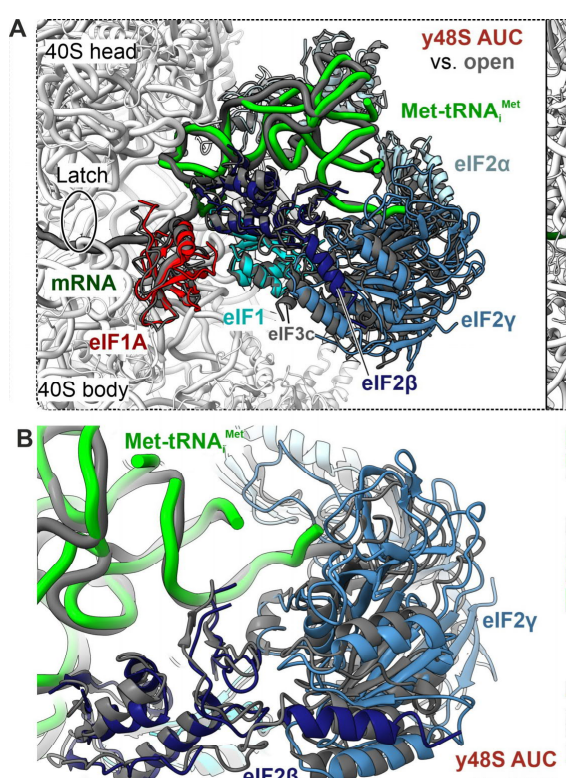
vs. open

E
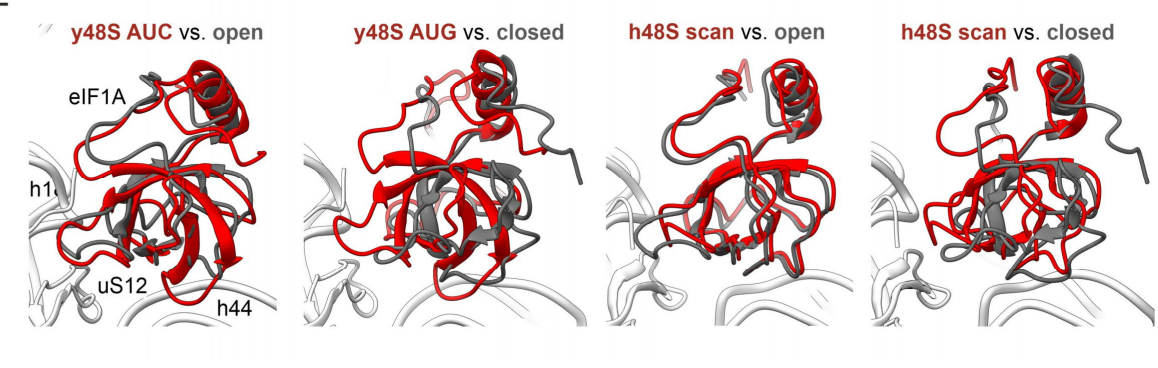

C
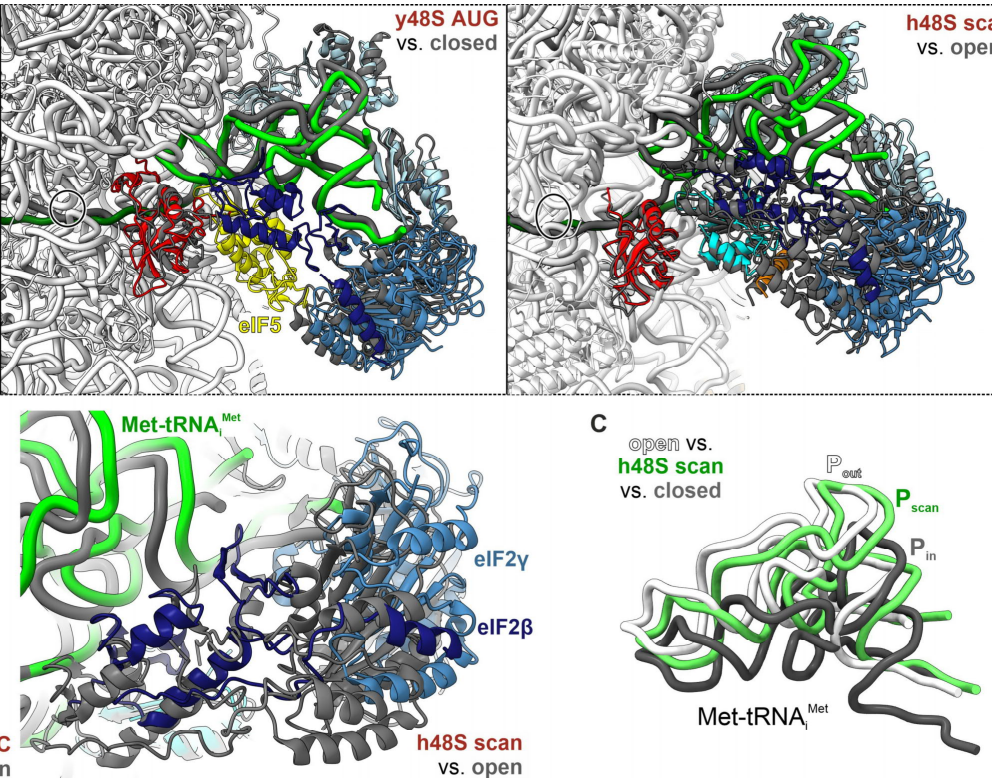
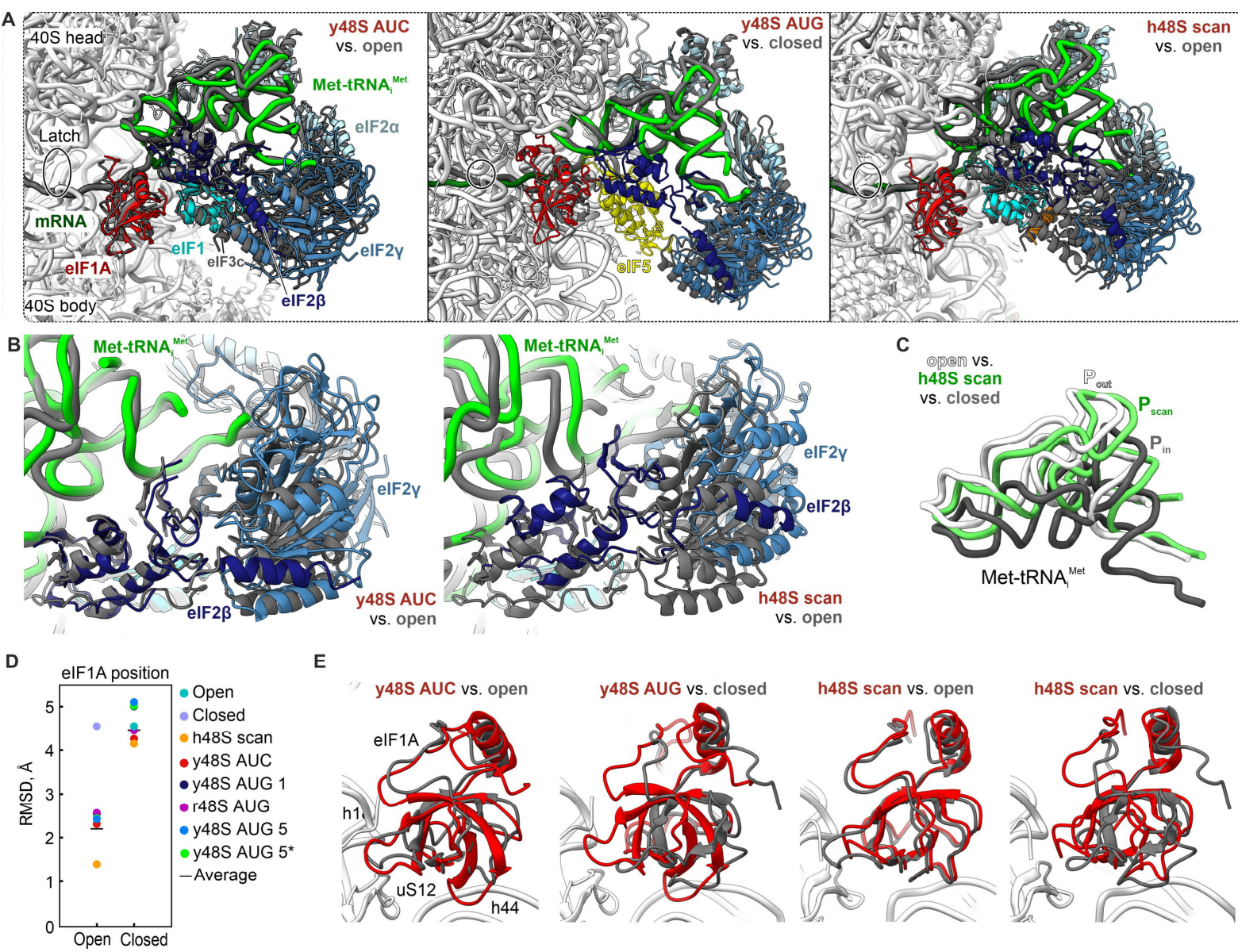

Figure 3. Structural dynamics of 48 IC.

(A) Superposition of h48S AUG open and closed with other reported 48S IC structures. h48S AUG: IFs and tRNA are shown in dark grey; for visual clarity, the 40S subunit was removed after alignment. In structures used for comparison, IFs and tRNA are shown in color, 40S subunit in white. Structures used for comparison are y48S open with near-cognate AUC start codon (y48S AUC, PDB 3JAQ, (Llacer et al., 2015)), y48S closed with elF5 bound (yellow) and cognate AUG start codon (y48S AUG, PDB 6FYX)) (Llacer et al., 2018), and h48S scan (PDB 6ZMW, (Brito Querido et al., 2020)). Note the stabilization of eIF $2 \beta$ in the decoding center of 48 S ICs with elF1 or elF5 bound. elF $2 \beta$ appears to dissociate from the decoding center in the closed h48S AUG IC, which has neither elF1 nor elF5 bound.

(B) Comparison of elF2 $\beta$ and tRNA body position and interactions in the present open state (dim grey) vs. reported $48 \mathrm{~S}$ ICs (colored).

(C) Position of initiator tRNA in the present open ( $\left.P_{\text {out }}\right)$ and closed $\left(P_{\text {in }}\right)$ states vs. the position in h48S scan $\left(P_{\text {scan }}\right)$.

(D) Unique rearrangement of elF1A in h48S AUG upon start codon recognition. Plot: Root-meansquare deviations (RMSDs) between the C $\alpha$-backbone of elF1A in the present h48S AUG open and closed states and the reported $48 \mathrm{~S}$ structures. y48S AUC, near-cognate yeast $48 \mathrm{~S} \mathrm{IC}$ with mRNA and elF1 bound (PDB 3JAQ, (Llacer et al., 2015)); y48S AUG 1, cognate yeast 48S IC with cognate mRNA 
and elF1 bound (PDB 3JAP, (Llacer et al., 2015)); y48S AUG 5, cognate yeast 48S IC with elF5 bound in state C1 (PDB 6FYX, (Llacer et al., 2018); y48S AUG 5*, cognate yeast 48S IC with elF5 bound in state C2 (PDB 6FYY, (Llacer et al., 2018)); r48S AUG, rabbit 48S IC with cognate $\beta$-globin mRNA (Simonetti et al., 2020); h48S scan, non-cognate human 48S IC (PDB 6ZMW, (Brito Querido et al., 2020)).

(E) Superposition of elF1A in present states (dim grey) vs. elF1A in reported 48 S ICs (red). Notably, the position of elF1A is similar in all 48S ICs, except the present closed h48S AUG IC, which adopts a substantially different position.

A
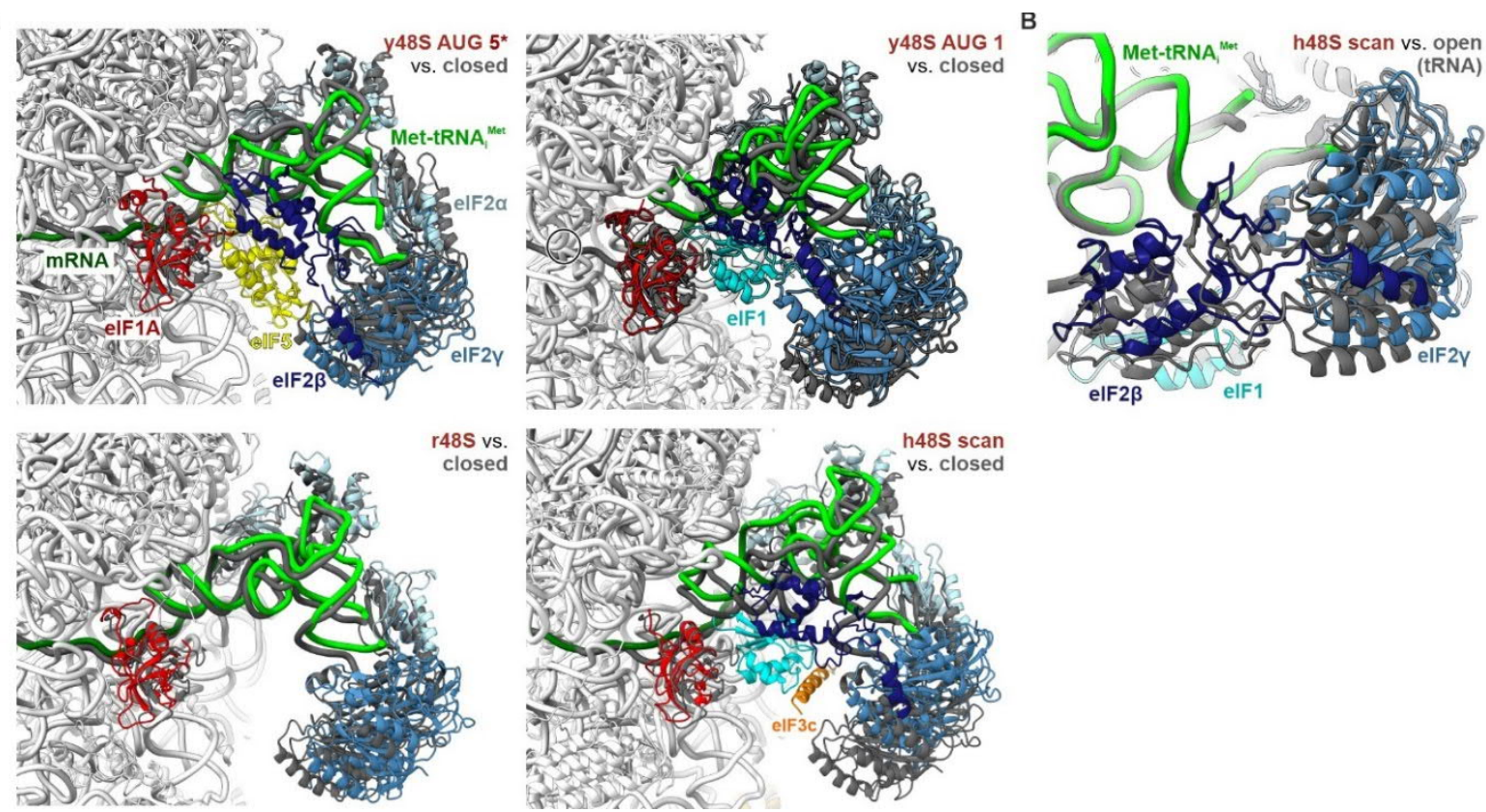

Figure 3 - figure supplement 1. Comparison of the decoding center and TC in 48S ICs

(A) Superposition of the h48S AUG structures (light grey, 40S omitted) with other reported 48S ICs. y48S AUG 5*, cognate yeast 48S IC with elF5 bound in state C2 (PDB 6FYY (Llacer et al., 2018)); y48S AUG 1, cognate yeast 48S IC with elF1 bound in state C1 (PDB 4JAP (Llacer et al., 2015)); r48S AUG, rabbit 48 S IC with cognate $\beta$-globin mRNA (Simonetti et al., 2020); h48S scan, non-cognate human 485 IC (PDB 6ZMW, (Brito Querido et al., 2020)).

(B) Close-up of elF2 $\beta$ and tRNA body. Alignment of h48S AUG open vs. h48S scan (PDB 6ZMW, (Brito Querido et al., 2020)).

Comparison between the structures of human $48 \mathrm{~S}$ complexes reveals that h48S AUG open and closed described here differ substantially from the h48S-scanning complexes (Brito Querido et al., 2020). The orientation of the $40 \mathrm{~S}$ head domain in h48S.scan (Figure 2 - figure supplement 1) renders partial opening of the decoding center and mRNA latch and a conformation of the TC distinct from open and closed states (Figures 2B, 3A and Figure 3 - 
figure supplement 1). Similar to the h48S AUG open, Met-tRNA ${ }_{i}{ }^{\text {Met }}$ in the scanning complex does not interact with the start codon (Figure 2B). However, due to the particular domain arrangement, the anticodon stem-loop of tRNA ${ }_{i}{ }^{\text {Met }}$ during scanning adopts a position half-way between $P_{\text {out }}$ and $P_{\text {in }}\left(P_{\text {scan }}\right)$ enabling formation of a contact with elF1 (Figure 3B,C, Figure 2D and Figure 3 - figure supplement 1 ). In comparison to the open state, the position of elF1 in h48S scan is slightly shifted. In both h48S AUG open and h48 scan, elF1 interacts with the Nterminal insertion of elF3c (Figure 2 - figure supplement 1C). In contrast, the helix-turn-helix domain of elF $2 \beta$ is found $10 \AA$ away from the decoding center in the scanning complex compared to the open complex. The TC, in particular elF $2 \alpha$ and elF $2 \gamma$, move in the same direction as elF2 $\beta$ and the contacts of elF2 $\beta$ with tRNA $_{i}{ }^{\text {Met }}$ are shifted from the anticodonstem loop in the open state to the $D$ loop region in the scanning complex (Figure $3 A, B$ ). In the closed state, elF $2 \alpha$ and elF $2 \gamma$ move in a different direction than in h48S scan and towards the $40 S$ body due to the simultaneous dissociation of elF $2 \beta$ from the decoding center and tRNA ${ }_{i}{ }^{M e t}$ docking onto the start codon (Figure 3 - figure supplement 1 ). The overall conformation of elF3 is very similar in the human $48 \mathrm{~S}$ complexes in the scanning, open and closed states, except for elF3j subunit, which is visible only in the scanning complex.

A major difference between h48S AUG closed and all other reported $48 \mathrm{~S}$ structures is the change in the position of elF1A (Figure 3D,E), with a root-mean-square deviation (RMSD) of about $4.5 \AA$ to any of these other structures. In the majority of structures, the position of elF1A is similar to that in our open h48S AUG PIC conformation, with an RMSD of only $2 \AA$. Considering the crucial role of elF1A in start site selection (Fekete et al., 2007; Llacer et al., 2018; Maag et al., 2006), the unique position of elF1A in our h48S AUG closed structure may represent an intermediate that forms after codon recognition, but prior to further steps on the path of 80 S IC assembly.

\section{S IC conformations probed by time-resolved fluorescence measurements}

Our findings showing the inherent structural heterogeneity of h48S AUG have prompted us to investigate the ribosome population distribution by time-resolved fluorescence methods. Following the previous work on y48S IC, we used elF1A as a reporter for conformational changes in the complex (Llacer et al., 2015; Llacer et al., 2018; Maag et al., 2006; Maag et al., 2005). elF1A is one of the first factors to bind to and one of the last to dissociate from the $40 \mathrm{~S}$ subunit upon 80 S IC formation (Acker et al., 2009; Fringer et al., 2007). Furthermore, the 
observed reorientation of eIF1A in h48S AUG closed (Figure 2F) is likely to change the local environment of elF1A, which can be used to monitor the 40 S subunit conformations using an environmentally sensitive fluorophore. To label different regions of elF1A, we introduced single cysteine residues at positions N4, S74 or T120, which are located at the N-terminal tail (NTT), oligonucleotide/oligosaccharide-binding (OB) domain, and the C-terminal tail (CTT) of elF1A, respectively (Figure 4A), and labeled these eIF1A variants with AlexaFluor555 (Alx555). All eIF1A derivatives are active in promoting $48 \mathrm{~S} \mathrm{IC} \mathrm{assembly} \mathrm{and} \mathrm{80S} \mathrm{EC} \mathrm{formation} \mathrm{as}$ indicated by toe-printing assay and peptide bond formation with efficiency similar to that in the presence of WT eIF1A (Figure 1 - figure supplement 2D and Figure 4 - figure supplement 1).

A

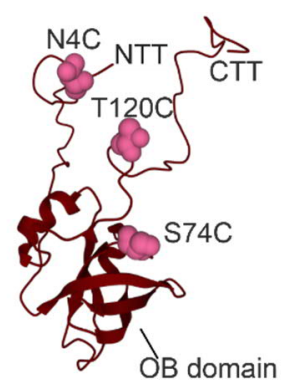

B

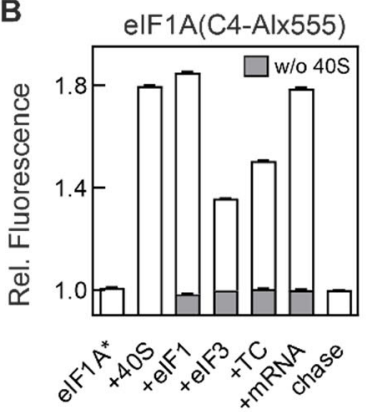

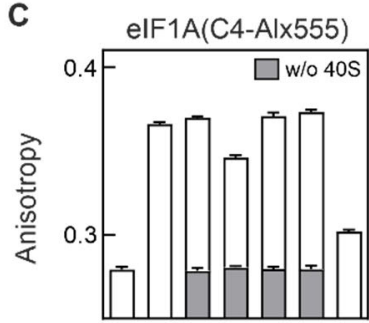

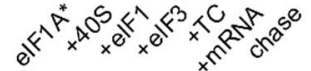

Figure 4. Monitoring the compositional and conformational dynamics of h48S using fluorescence-labeled elF1A(C4-Alx555).

(A) Structure of eIF1A (PDB 1D7Q; (Battiste et al., 2000)). Alexa 555-labeling positions (pink) are shown in the NTT, the OB domain, and the CTT of elF1A.

(B), (C) Relative fluorescence intensity (B) and anisotropy (C) changes of elF1A(C4-Alx555). 48S IC was assembled by sequential addition of factors as indicated. In (B), fluorescence intensity of free elF1A(C4-Alexa555) is set to 1.0. Fluorescence intensity and anisotropy measured in the absence of the $40 \mathrm{~S}$ subunit (w/o 40S) are shown in dark grey. Error bars represent standard deviations of 5 technical replicates $(\mathrm{N}=5)$. 


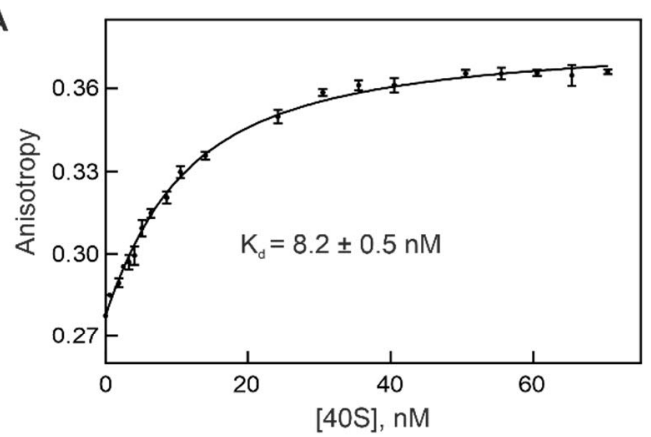

C

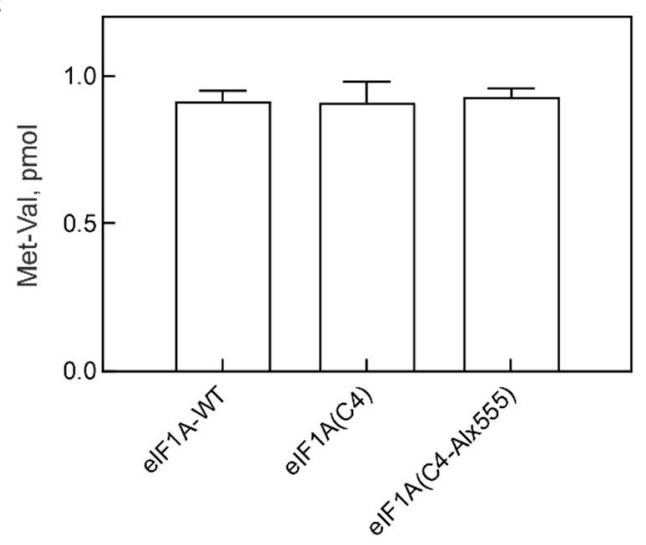

B

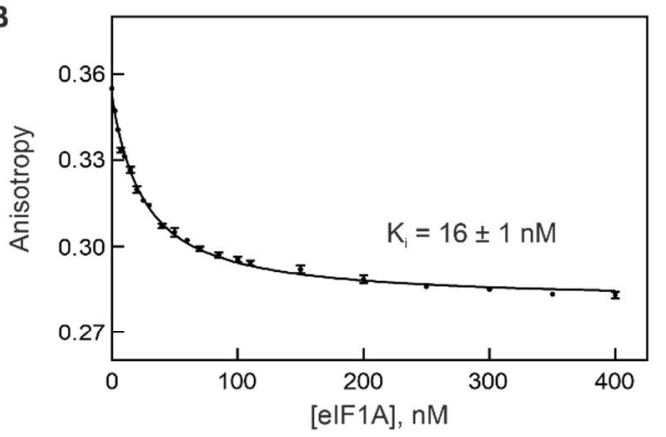

Figure 4 - figure supplement 1 . Functional activity of elF1A(C4-Alx555).

(A) Affinity of elF1A(C4-Alx555) binding to the 40S subunit. elF1A(C4-Alx555) (5 nM) was titrated with increasing concentrations of the $40 \mathrm{~S}$ subunit at $25^{\circ} \mathrm{C}$. Anisotropy of elF1A(C4-Alx555) was measured in a spectrofluorometer with excitation at $555 \mathrm{~nm}$ and emission at $568 \mathrm{~nm}$. The binding curve was evaluated using a quadratic equation to obtain the binding affinity $\left(K_{d}\right)$.

(B) Comparison of the binding affinities of fluorescence-labeled and unlabeled elF1A using a competition titration assay. The 40S-elF1A complex (5 $\mathrm{nM})$ was titrated with increasing concentrations of unlabeled elF1A. The $\mathrm{IC}_{50}$ of the inhibition curve and the $\mathrm{K}_{\mathrm{i}}$ value (which reflects the $K_{d}$ for unlabeled elF1A) were calculated using GraphPad prism software.

(C) Met-Val dipeptide formation on 80S EC assembled using elF1A(wt), elF1A(C4) and elF1A(C4Alx555). 80 S IC ( 2 pmol) was assembled with the respective elF1A variant and mixed with a 10 -fold excess of eEF1A-GTP-[ $\left[{ }^{14} \mathrm{C}\right]$ Val-tRNA ${ }^{\mathrm{Val}}$. The Met-Val dipeptide was analyzed by HPLC and quantified using $\left[{ }^{14} \mathrm{C}\right]$ radioactivity counting in the Met-Val and Val peaks. 
A

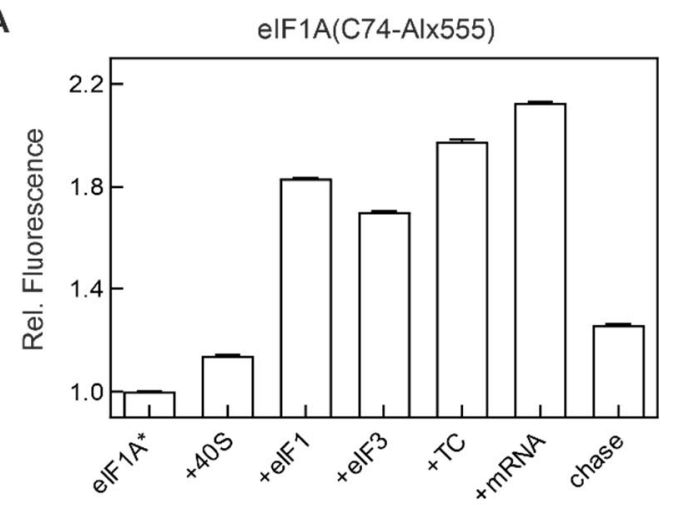

B

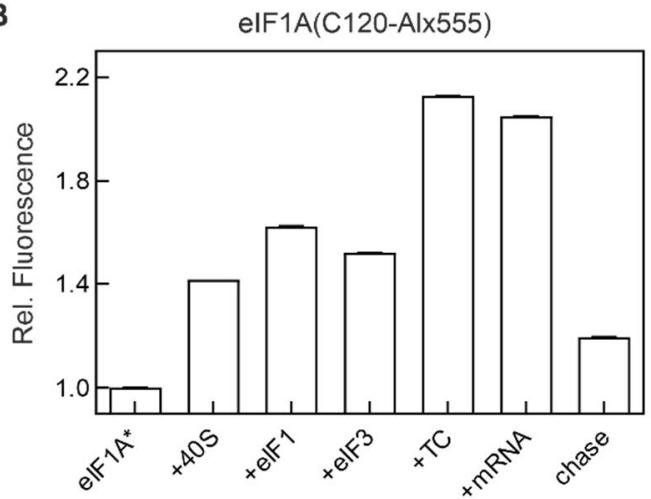

Figure 4 - figure supplement 2. Fluorescence intensity changes of elF1A(C74-Alx555) and elF1A(C120-Alx555) upon 48S IC formation.

(A) eIF1A(C74-Alx555). (B) eIF1A(C120-Alx555). Components of 48S IC were added sequentially in the order as indicated. Fluorescence intensity of free labeled elF1A was set to 1.0. Error bars show standard deviation of 5 technical replicates $(\mathrm{N}=5)$.

We first tested whether the fluorescence intensity of labeled elF1A is sensitive to compositional changes at different steps of 48 S IC assembly. Each one of the labeled elF1A derivatives shows a distinct fluorescence signature upon binding to the 405 subunit and subsequent stepwise addition of elFs (Figure 4B and Figure 4 - figure supplement 2). The magnitude of the fluorescence change depends on the labeling position. For example, fluorescence of elF1A(C4-Alx555) increases by $80 \%$ upon binding to the 40 s subunit and is not further altered by elF1 recruitment, but decreases considerably upon binding of elF3 (Figure 4B). Addition of TC has only a small effect. Upon binding of mRNA with an AUG start codon, fluorescence intensity increases again, consistent with the expected rearrangement upon start codon recognition. Chase of elF1A(C4-Alx555) with excess amount of unlabeled elF1A restores the fluorescence value before binding to the $40 \mathrm{~S}$ subunit, demonstrating that the signal results from a reversible interaction of elF1A with the $40 \mathrm{~S}$ subunit. In the absence of the $40 \mathrm{~S}$ subunit, the fluorescence of elF1A(C4-Alx555) does not change when other elFs are added, showing that the observed fluorescence changes reflect the $48 \mathrm{~S} \mathrm{IC} \mathrm{assembly} \mathrm{(Figure}$ 4B). The fluorescence profiles of elF1A(C74-Alx555) and elF1A(C120-Alx555) are somewhat different from elF1A(C4-Alx555), e.g., elF1A(C74-Alx555) is particularly sensitive to elF1 recruitment, whereas eIF1A(C120-A555) monitors 40S subunit and TC recruitment (Figure 4 - figure supplement 2). We further validated the fluorescence intensity approach by measuring anisotropy of elF1A(C4-Alx555) (Figure 4C). The addition of 40 S subunit to 
elF1A(C4-A555) leads to an anisotropy increase due to formation of a stable 40S-elF1A complex. Addition of elFs and mRNA does not alter anisotropy, indicating that elF1A remains bound to the 40 S subunit throughout the 48 S IC assembly. A small, but significant, anisotropy decrease with elF3, together with the pronounced decrease of fluorescence intensity suggests that elF3 binding to the 40S-elF1A-elF1 complex loosens elF1A binding; this effect is reversed upon TC binding. Anisotropy decreases only when dissociation of elF1A(C4-Alx555) is induced by the addition of excess unlabeled elF1A (chase) or upon 80 S formation induced by addition of elF5B and the 60S subunit (see below), in agreement with the notion that elF1A dissociates during 60S subunit joining (Acker et al., 2009; Fringer et al., 2007). In the following, we used the changes in eIF1A fluorescence intensity to follow structural and compositional rearrangements upon assembly of translation initiation complexes.

\section{h48S rearrangements on the pathway to start codon selection}

Previous work on yeast initiation has shown that elF1A dissociation kinetics is indicative of $40 S$ subunit conformations (Llacer et al., 2018; Maag et al., 2006; Maag et al., 2005), which prompted us to use a similar approach to study how codon recognition affects the complex conformation in the mammalian system. We first measured the dissociation rates of fluorescence-labeled elF1A from h48S CUC, h48S AUC and h48S AUG complexes upon mixing h48S with excess unlabeled elF1A in a stopped-flow apparatus (Figure 5A and Table 1). To avoid potential bias due to the labeling position, we carried out these experiments with three elF1A derivatives labeled at different sites of the protein, which showed very similar effects (Figure 5B-F and Table 1). Dissociation of elF1A from the h43S PIC, h48S CUC and h48S AUC follows single exponential kinetics, with the rates of $0.01 \mathrm{~s}^{-1}, 0.007 \mathrm{~s}^{-1}$, and $0.03 \mathrm{~s}^{-1}$, respectively. The dissociation is slow on a physiological scale, consistent with the notion that elF1A remains bound to the $40 \mathrm{~S}$ subunit until the $60 \mathrm{~S}$ subunit joining (Acker et al., 2009; Fringer et al., 2007). In the simplest model, the single exponential kinetics suggests that the major contacts of elF1A are similar on most of $40 \mathrm{~S}$ subunits in the population, i.e. the h43S PIC and h48S PICs representing the scanning (h48S CUC) or partial codon reading (h48S AUC) comprise homogeneous populations with respect to the decoding center conformation. In contrast, chase of elF1A from h48S AUG shows two-exponential dissociation kinetics (Figure $5 E, F$ and Table 1), indicating the presence of two distinct populations of complexes. The slow dissociation $\left(0.005 \mathrm{~s}^{-1}\right)$ from h48S AUG is in the same range as from scanning h48S CUC. The 
additional kinetic phase has a much higher rate $\left(0.11 \mathrm{~s}^{-1}\right)$ and appears only in the presence of the cognate AUG codon, suggesting that start codon recognition induces rapid dissociation of elF1A from a population of h48S IC. The presence of two distinct h48S AUG populations is consistent with the cryo-EM reconstructions presented above (Figure 1). It is therefore likely that the kinetic population with a rapid eIF1A dissociation that appears upon AUG codon recognition represents h48S AUG closed that is predominant in the cryo-EM sample and yields stable $48 \mathrm{~S} \mathrm{IC} \mathrm{in} \mathrm{the} \mathrm{toe-printing} \mathrm{analysis} \mathrm{(Figure} 1$ - figure supplement $2 \mathrm{C}$ ), whereas the population that releases elF1A more slowly corresponds to h48S AUG open population.

A
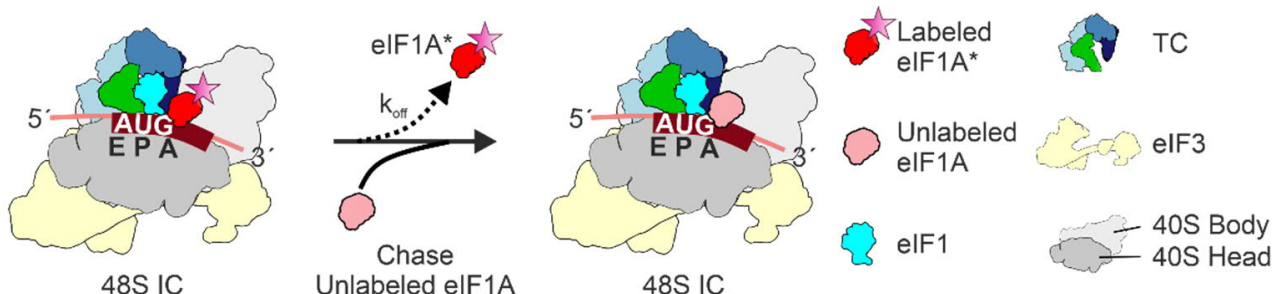

B

$43 S$
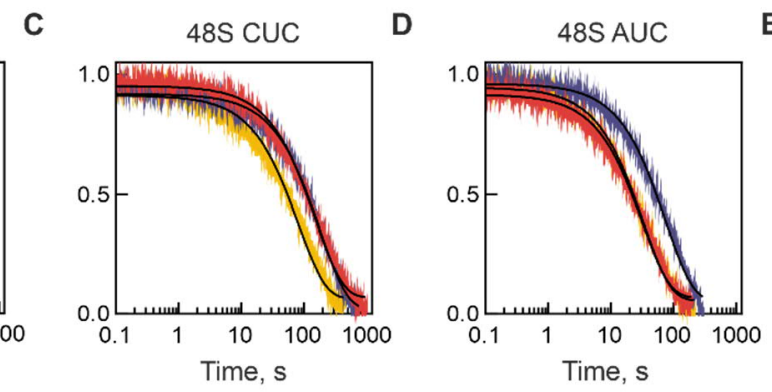

$\mathrm{E}$

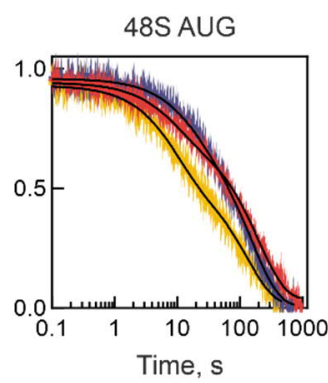

F

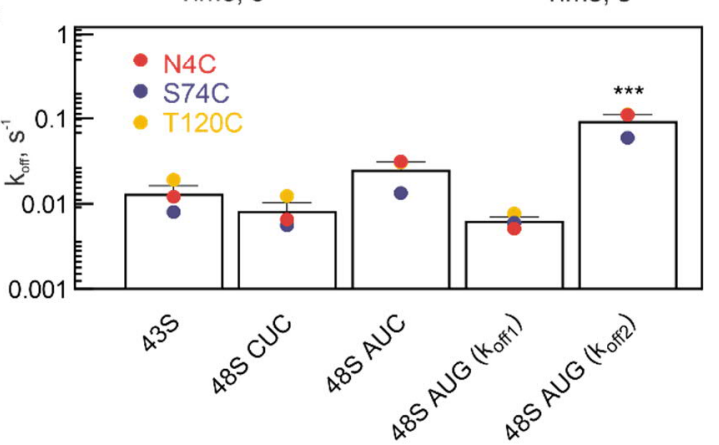

\section{Figure 5. Dynamics of 48S IC upon start codon selection.}

(A) Schematic of the chase experiment. The 43S PIC or 48S IC (15 nM) assembled from 40S subunits with eIF1, TC, eIF3, eIF4A, elF4B, fluorescence-labeled $\left({ }^{*}\right)$ eIF1A, with or without mRNA was rapidly mixed with excess of unlabeled elF1A $(1.8 \mu \mathrm{M})$ in the stopped-flow apparatus.

(B-E) Time courses of elF1A dissociation from 43S PIC (B), 48S CUC (C), 48S AUC (D) and 48S AUG IC (E) measured using elF1A labeled at different positions. Black lines show results of one-exponential (B-D) 
or two-exponential (E) fitting. Fluorescence intensity change was normalized to 0-1 range. Time courses represent averages of 5 technical replicates $(\mathrm{N}=5)$.

(F) Summary of the elF1A dissociation rates from different initiation complexes (B-E). Bar graphs show average values from three different reporters with the standard deviation $(\mathrm{N}=3)$. For each reporter, the standard deviation of the measurement is smaller than the symbol size. ${ }^{* * *}{ }^{*}$ indicates that the $\mathrm{k}_{\text {off2 }}$ of $48 \mathrm{~S} \mathrm{IC} \mathrm{(AUG)} \mathrm{measured} \mathrm{with} \mathrm{each} \mathrm{elF1A} \mathrm{variant} \mathrm{is} \mathrm{significantly} \mathrm{different} \mathrm{from} \mathrm{other} \mathrm{k}_{\text {off }}$ values for the same variant as compared using a two-tailed t-test ( $P$-value $<0.001)$. Note the logarithmic scale of the $Y$-axis.

Table 1. Summary of elF1A dissociation rates from h43S PIC and various h48S complexes

\begin{tabular}{|c|c|c|c|c|c|}
\hline $\begin{array}{l}\text { Initiation } \\
\text { complex }\end{array}$ & mRNA & elFs & $\begin{array}{c}\text { elF1A } \\
\text { derivative }\end{array}$ & $k_{\text {off1 } 1}, s^{-1}$ & $k_{\text {off2 }}, s^{-1}$ \\
\hline \multirow[t]{3}{*}{$43 S$ PIC } & - & - & C4 & $0.01200 \pm 0.00004$ & \\
\hline & & & $\mathrm{C} 74$ & $0.00800 \pm 0.00004$ & \\
\hline & & & C120 & $0.01900 \pm 0.00009$ & \\
\hline \multirow[t]{3}{*}{ 48S PIC } & CUC & - & C4 & $0.00654 \pm 0.00003$ & \\
\hline & & & $\mathrm{C} 74$ & $0.00556 \pm 0.00003$ & \\
\hline & & & C120 & $0.01231 \pm 0.00008$ & \\
\hline \multirow[t]{3}{*}{ 48S PIC } & AUC & - & C4 & $0.0312 \pm 0.0001$ & \\
\hline & & & $\mathrm{C} 74$ & $0.01335 \pm 0.00005$ & \\
\hline & & & C120 & $0.0304 \pm 0.0001$ & \\
\hline \multirow[t]{3}{*}{$48 \mathrm{~S} \mathrm{IC}$} & AUG & - & C4 & $0.00510 \pm 0.00003$ & $0.110 \pm 0.001$ \\
\hline & & & $\mathrm{C} 74$ & $0.00600 \pm 0.00003$ & $0.0590 \pm 0.0008$ \\
\hline & & & C120 & $0.00770 \pm 0.00007$ & $0.1110 \pm 0.0009$ \\
\hline \multirow[t]{7}{*}{$48 \mathrm{~S} \mathrm{IC}$} & AUG & w/o elF1 & $\mathrm{C} 4$ & $0.0190 \pm 0.0001$ & $0.2680 \pm 0.002$ \\
\hline & & w/o TC & $\mathrm{C} 4$ & $0.0440 \pm 0.0001$ & \\
\hline & & w/o elF3 & C4 & $0.01900 \pm 0.00007$ & \\
\hline & & +elF5 & C4 & $0.0165 \pm 0.0001$ & $0.153 \pm 0.002$ \\
\hline & & +elF5B & C4 & $0.00361 \pm 0.00001$ & \\
\hline & & +elF5+elF5B & $\mathrm{C} 4$ & $0.00275 \pm 0.00001$ & \\
\hline & & w/o elF1 + elF5B & C4 & $0.00396 \pm 0.00001$ & \\
\hline
\end{tabular}

43S PIC contains 40S subunits, elF1, elF1A, TC, elF3, elF4A, and elF4B. 48S IC is assembled from 40S subunits, elF1, elF1A, TC, elF3, elF4A, elF4B, and mRNA, if not stated otherwise. mRNA contains an optimal Kozak sequence with the AUG codon or CUC or AUC codons replacing the AUG.

Our results suggest two interesting differences to the yeast system, where similar experiments were performed by measuring anisotropy of fluorescence-labeled elF1A. First, elF1A dissociation from y48S complex is biphasic irrespective of the initiation start codon. Second, AUG recognition results in a preferential stabilization of elF1A on y48S IC (Llacer et 
al., 2018; Maag et al., 2006; Maag et al., 2005), suggesting that start codon recognition in yeast leads to tighter binding of elF1A, rather than weaker binding which we find in the human system. In part, this may be due to the compositional differences between y48S IC and our h48S IC, as yeast complexes used in kinetic studies were mostly assembled in the absence of elF3. This is possible, because elF3 is dispensable for translation initiation on model mRNAs in yeast, but not feasible in mammalian system, where elF3 is essential for h48S IC formation (Figure 1 - figure supplement 2B) (Majumdar et al., 2003; Pestova and Kolupaeva, 2002). In addition, y48S IC was assembled in the presence of elF5, which is dispensable for h48S IC assembly (Figure 1 - figure supplement 2A,B)(Pestova and Kolupaeva, 2002). These differences prompted us to further investigate how the ribosome population distribution and elF1A dissociation depend on the set of elFs bound to h48S complexes.

\section{elFs modulate ribosome population distribution}

First, we followed elF1A dissociation from h48S IC lacking one of the essential elFs. In the absence of TC or eIF3, eIF1A release is relatively fast and the time courses are single exponential, indicating a uniform ribosome conformation with respect to elF1A binding. In the mammalian system, TC and elF3 are both required for start codon recognition, and in their absence, h48S PIC does not form a stable complex on the AUG codon, as indicated by the lack of the respective characteristic band in the toe-printing assay (Figure 1 - figure supplement 2B). TC stabilizes elF1A binding on the 43S PIC in both yeast and mammals (Algire et al., 2002; Maag et al., 2005; Sokabe and Fraser, 2014), which explains why elF1A dissociation is faster in the absence than in the presence of TC (Figure 6A,C and Table 1). elF3 may affect elF1A binding indirectly, e.g. by stabilizing elF1 in the open state of the 40S subunit (Figure 3 - figure supplement 1A) (Majumdar et al., 2003; Sokabe and Fraser, 2014), which would also explain why the lack of these interactions results in a more rapid elF1A dissociation. 
A

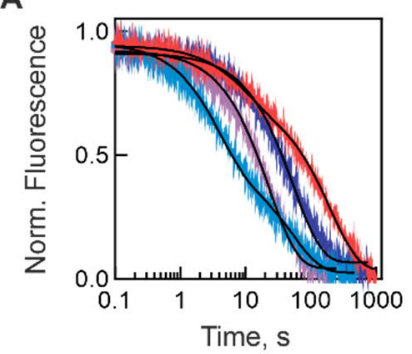

$48 S$ IC

$48 \mathrm{~S}$ w/o TC

$48 \mathrm{~S}$ w/o elF3

$48 \mathrm{~S}$ w/o elF1

C

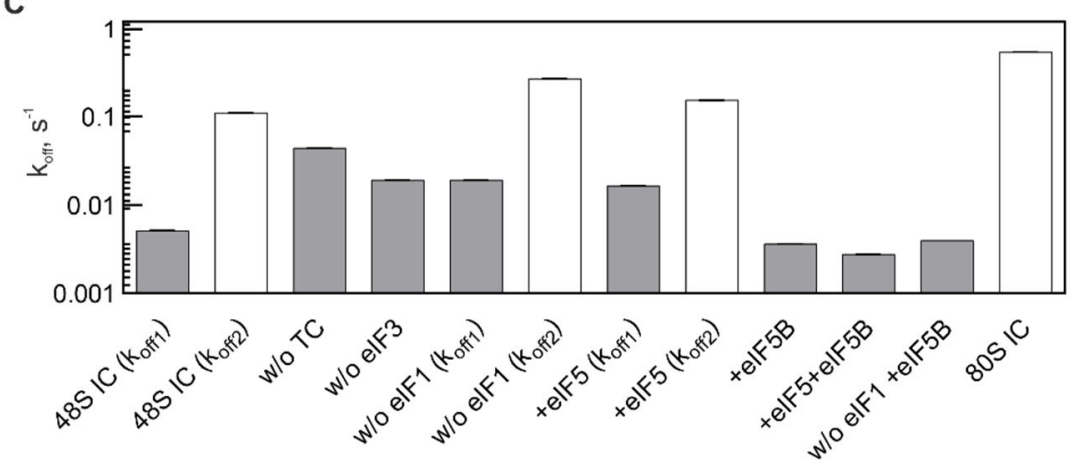

B

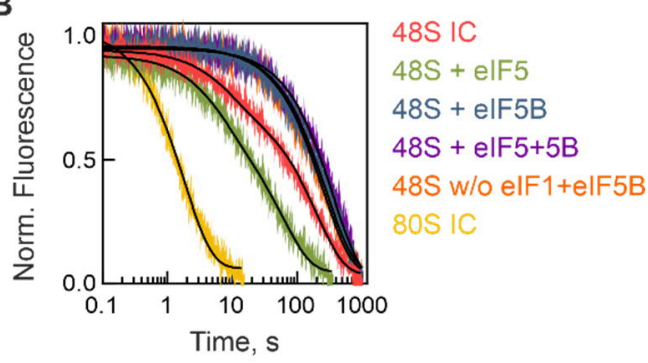

Figure 6. Dynamics of h48S IC.

(A) Time-courses of elF1A dissociation from the partial h48S IC lacking elF1, TC or elF3. Fitting of the time courses was single-exponential for complexes lacking TC or elF3 and two-exponential for h48 IC with or without elF1. Fluorescence change is normalized to 0-1 range. Time courses are averages of five technical replicates $(\mathrm{N}=5)$.

(B) Time-courses of elF1A dissociation from $48 \mathrm{~S} \mathrm{IC} \mathrm{in} \mathrm{the} \mathrm{presence} \mathrm{of} \mathrm{elF5} \mathrm{or} \mathrm{elF5B} \mathrm{or} \mathrm{from} \mathrm{80S} \mathrm{IC.}$ Dissociation of elF1A from the 80 S complex was induced by mixing 60 S subunits with the 48 S AUG in the presence of elF5 and elF5B. Time courses in the presence of elF5 were analyzed by twoexponential fitting; those with elF5B, elF5 and elF5B, w/o elF1+elF5B, and 80S IC were analyzed by one-exponential fitting. Fluorescence change is normalized to 0-1 range. Time courses are averages of five technical replicates $(\mathrm{N}=5)$.

(C) Summary of the $k_{\text {off }}$ values. Rates of single-exponential reactions as well as slow phases of twoexponential time courses are shown in grey, the fast phases and dissociation from 805 in white bars. Error bars (very small) show standard deviation of five technical replicates $(\mathrm{N}=5)$. 
In contrast to other factor omission experiments, eIF1A dissociation from h48S AUG lacking elF1 is biphasic and both dissociation rates are somewhat faster than from the complete h48S IC (Figure 6A,C and Table 1). The increase in the dissociation rates is expected, because elF1 and elF1A are known to stabilize each other's binding to the 40S subunit (Maag and Lorsch, 2003; Majumdar et al., 2003; Pestova et al., 1998; Sokabe and Fraser, 2014). Notably, we found a stable 48S IC toe-print on the AUG codon in the absence of elF1 (Figure 1 - figure supplement 2D). This is consistent with the notion that elF1A can compensate for the lack of elF1 during 48S IC assembly on a model mRNA with an optimal initiation start site, as used in this study (Fekete et al., 2007; Pestova and Kolupaeva, 2002). This also explains why h48S IC lacking elF1 can recognize the AUG codon, leading to formation of the characteristic twopopulation distribution of the $40 \mathrm{~S}$ subunits in the complex (Figure $6 \mathrm{~A}, \mathrm{C}$ and Table 1 ).

Next, we studied the effect of elF5 and elF5B (Figure 6B,C and Table 1). elF1A dissociation from h48S AUG in the presence of elF5 is biphasic and the dissociation rates are comparable to those from h48S IC without elF1 (Fig 6A,C and Table 1). The latter is in agreement with the finding that elF5 displaces elF1 upon start codon recognition (Llacer et al., 2018), but the observed destabilization of elF1A kinetics on h48S AUG is at variance to the yeast system, where eIF1A binds more tightly upon elF5 addition (Llacer et al., 2018; Maag et al., 2006; Maag et al., 2005). Finally, addition of elF5B stabilizes the h48S AUG in a single major population with tight eIF1A binding (Figure $6 \mathrm{~B}, \mathrm{C}$ and Table 1). elF5B compensates the destabilizing effect of elF5 and reverses the elF1 omission effect, suggesting that elF5B acts after the remodeling of the decoding center by elF5. The h48S IC with elF5B bound is the last intermediate on the pathway of translation initiation before the 60S subunit docking, which remodels the complex to an 80 S IC ready to start translation. The 605 subunit joining triggers fast release of eIF1A from the $80 \mathrm{~S} \mathrm{IC} \mathrm{with} \mathrm{a} \mathrm{rate} \mathrm{of} 0.53 \mathrm{~s}^{-1}$. Overall, these results support the notion that the two-population distribution of h48S AUG is characteristic for complexes upon start codon recognition in the mammalian system. 


\section{Discussion}

The results of our experiments suggest how start codon recognition modulates the structure of human h48S IC (Figure 7). In h43S PIC, which we characterize by kinetic experiments using elF1A as reporter, $40 \mathrm{~S}$ subunit is found in a single predominant conformation that binds elF1A tightly. This characteristic state of the complex is maintained throughout mRNA scanning. Partial recognition of the near-cognate codon AUC by Met-tRNA ${ }_{i}^{\text {Met }}$ has a small destabilizing effect on elF1A binding. Start codon recognition changes the conformational distribution in the ensemble, and both kinetic and structural studies consistently show two conformations of h48S AUG. The structure of the open h48S AUG PIC shows a small fraction of complexes that do not undergo codon-anticodon interaction and bind elF1A tightly. On the majority of ribosomes, however, start codon recognition induces structural remodeling of the decoding site that induces $40 \mathrm{~S}$ domain closure, movement of Met-tRNA ${ }_{i}{ }^{\text {Met }}$ into the $\mathrm{P}_{\text {in }}$ conformation, displacement of elF1 and eIF2 $\beta$, as well as a rearrangement of elF1A that results in faster dissociation rate of elF1A from the ribosome. Binding of elF5 has an additional, albeit small, destabilizing effect, but the resulting complexes still comprise two kinetic populations. Recruitment of elF5B, which is a prerequisite for the efficient 60S subunit docking, induces a rearrangement to a predominant $40 \mathrm{~S}$ conformation to which elF1A binds tightly, potentially owing to a direct contact between elF5B and eIF1A (Acker et al., 2006; Acker et al., 2009; Lin et al., 2018). Finally, elF1A dissociates rapidly from the 80 S IC, probably following GTP hydrolysis by elF5B (Acker et al., 2009; Lee et al., 2002; Pestova and Kolupaeva, 2002; Pestova et al., 2000) after 60S joining (Figure 7).

The structure of h48S AUG open differs from the scanning conformation of h48S (Brito Querido et al., 2020), suggesting that the two states are functionally distinct or at least represent different snapshots at the scanning pathway. In both structures, the codonanticodon interaction is not established, but the $40 \mathrm{~S}$ head domain is tilted to a different degree, resulting in a shift of Met-tRNA ${ }_{i}^{\text {Met }}$ position. On the other hand, open h48S AUG PIC is similar to y48S AUC, except for the partial codon-anticodon interaction in the near-cognate yeast complex, which is not found in h48S AUG open. Because h48S AUG open contains a full complement of elFs required to form h48S IC, this intermediate is unlikely to be an inactive off-pathway complex. With these comparisons in mind, we suggest that h48S AUG open represents ribosomes that attempt to read the start codon, but before the tRNA succeeds to form the first base-pair of the stable codon-anticodon complex. The observed 405 domain 


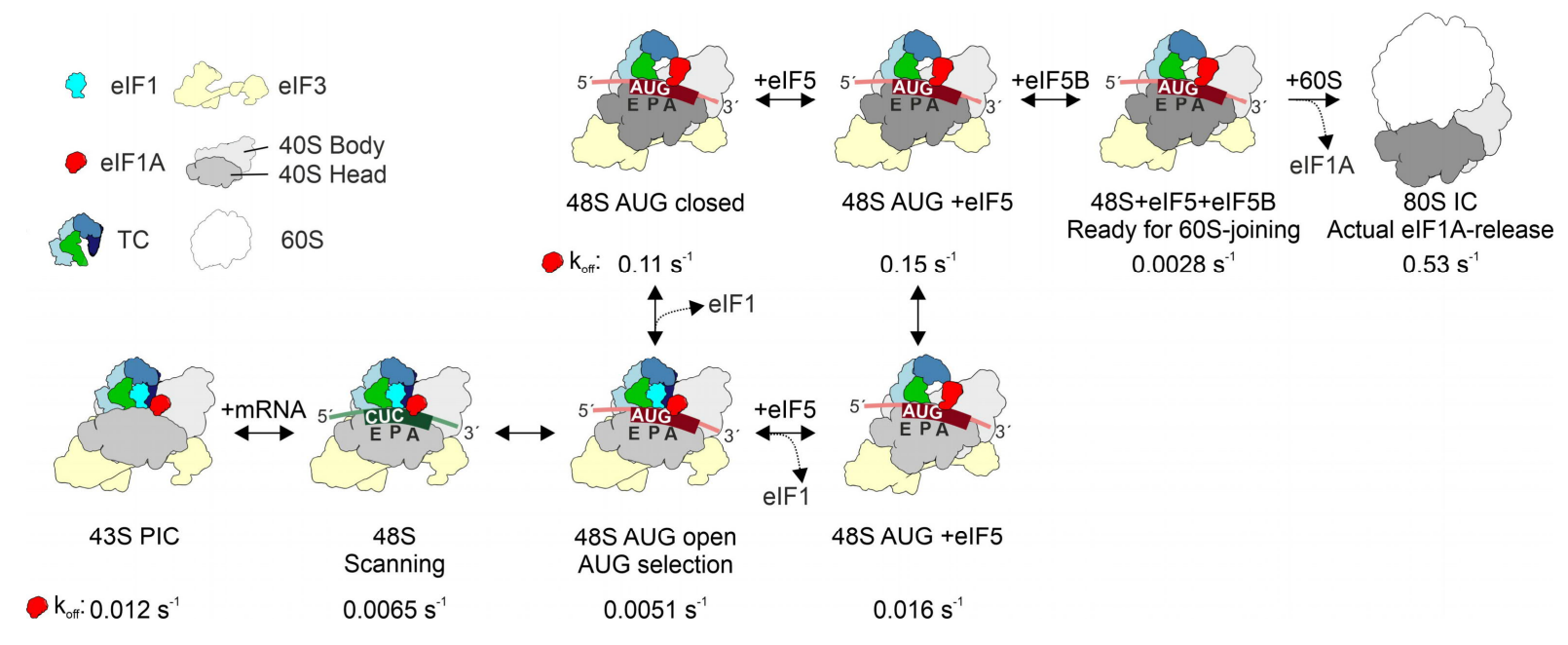

Figure 7. Kinetic model of late-stage human $48 \mathrm{~S}$ IC assembly.

43S PIC and 48S scanning complex comprise uniform populations as monitored by elF1A dissociation kinetics; elF1A dissociation rates ( $k_{\text {off }}$ ) are indicated. In contrast, h48S AUG complexes comprise two populations that correspond to h48S AUG open (prior to codon recognition, with low $k_{\text {off }}$ for elF1A, labeled with light 40S head) and h48S AUG closed (after codon recognition, with high koff for elF1A, labeled with dark $40 \mathrm{~S}$ head). Upon codon recognition, elF1 is displaced, which can occur spontaneously (as indicated by the cryo-EM structures in this paper) or facilitated by elF5. In the mammalian system, these rearrangements depend on elF3. A partial codon recognition complex akin to that formed in y48S AUC has a low elF1A dissociation rate $\left(0.03 \mathrm{~s}^{-1}\right)$ and may transiently form also upon AUG recognition, but is not captured by structural studies and therefore not indicated in the scheme. Binding of elF5B stabilizes a single population which binds elF1A tightly. Finally, upon $60 \mathrm{~S}$ joining, elF1A rapidly dissociates from $80 \mathrm{~S} \mathrm{IC}$. The binding sites of elF5 and elF5B are not shown for visual clarity. The $k_{\text {off }}$ values in the schematic are derived from measurements with elF1A(C4-Alx555).

opening compared to the scanning conformation may facilitate the accommodation of MettRNA ${ }_{i}{ }^{M e t}$ and $m R N A$ in the decoding center. Thus, if partial AUC codon recognition complexes are similar in yeast and human systems, a likely order of complexes on the translation initiation pathway is 43S PIC -> scanning h48S CUC PIC ->h48S AUG open PIC -> partial codon recognition 48S AUC PIC -> closed h48S AUG IC (Figure 7). We note, however, that there are kinetic differences between the yeast and human $43 \mathrm{~S}$ and $48 \mathrm{~S}$ PICs, as revealed by the elF1A dissociation assay. While h43S PIC, as well as the scanning and partial codon recognition h48S PIC comprise a single major kinetic population, all yeast complexes entail two populations 
(Llacer et al., 2018; Maag et al., 2006; Maag et al., 2005). The structural basis for the two distinct conformations in Y48S PIC is not clear, as structural studies reveal a single major ribosome population of y48S AUC PIC or y48S AUG IC, possibly due to the use of the tRNA ${ }_{i}^{\text {Met }}$ mutant that stabilized the $P_{\text {in }}$ state (Llacer et al., 2015).

The population of the ribosomes that have formed stable codon-anticodon interactions adopted a closed conformation. They are structurally similar to other reported $48 \mathrm{~S} \mathrm{IC}$ complexes from yeast and mammals (Eliseev et al., 2018; Llacer et al., 2015; Llacer et al., 2018; Simonetti et al., 2020). Surprisingly, the presence of elF1A in r48S IC appears to depend on the type of the mRNA, e.g., the complex with the histone $\mathrm{H} 4$ mRNA displays no elF1A density, whereas the complex with $\beta$-globin mRNA shows elF1A in a state similar to that in our open h48S AUG state (Simonetti et al., 2020). Several other structures of mammalian complexes lacking one or more of the essential factors have been reported, but are not discussed here (Eliseev et al., 2018; Simonetti et al., 2016) (Simonetti et al., 2020).

The destabilizing effect of start codon recognition on elF1A binding to h48S AUG IC is at odds with the previous results obtained in the yeast system, where codon-recognition was shown to increase the binding stability of elF1A. One potential explanation would be the presence of elF5 in yeast complexes, as we initially did not use elF5 to assemble h48S AUG IC. We note that potential elF5-dependent effect would be due to elF5 binding, rather than GTP hydrolysis, as kinetic experiments in the yeast system were carried out in the presence of a non-hydrolysable GTP analog (Llacer et al., 2018; Maag et al., 2006). Our complexes contain GTP, which is hydrolyzed only upon addition of elF5 (Majumdar and Maitra, 2005; Unbehaun et al., 2004); thus, in both h48S AUG and y48S AUG, elF2 is expected to be in the GTP-bound form. Our kinetic measurements suggest that in the human system addition of elF5 (or omitting elF1, which should mimic the remodeling effect of elF5 on the decoding center) did not stabilize elF1A binding on either of the two ribosome populations. These findings suggest that the difference in kinetic stability of eIF1A in the mammalian vs. yeast system is not due to the presence or absence of elF5, but must depend on other factors, e.g. the presence of elF3 or the details of interactions that are difficult to discern at the current resolution of existing cryo-EM structures. In mammals, elF1 stabilizes the binding of elF1A and inhibits premature GTP hydrolysis by elF2. AUG recognition relieves this inhibition due to replacement of elF1 by elF5 and thereby destabilizes elF1A binding. Thus, the opposite effects 
of codon recognition on the stability of eIF1A binding in mammals and yeast may be related to the different ways by which elF1 controls the GTPase activity of elF2 in these organisms.

Another difference to the yeast system concerns the effect of elF3. In yeast, the elF1A binding is independent of the presence of elF3 (Maag et al., 2006). In contrast, in the mammalian system eIF3 is essential for h48S IC formation (Kumar et al., 2016; Pestova and Kolupaeva, 2002; Sokabe and Fraser, 2014; Sokabe et al., 2012). elF3 together with elF1 stabilize eIF1A binding to the $40 \mathrm{~S}$ subunit and are essential for recruiting the TC to the $43 \mathrm{~S}$ PIC and for scanning (Kumar et al., 2016; Majumdar et al., 2003; Pestova and Kolupaeva, 2002; Sokabe and Fraser, 2014). elF3 contacts the mRNA 5' UTR upstream of the start codon and protects $17 \mathrm{nt}$ of the mRNA in the vicinity of the mRNA exit channel in h48S IC (Pisarev et al., 2008). Interactions of elF3c with elF1, and at the later initiation steps with elF5, are important for start site selection (Obayashi et al., 2017; Valasek et al., 2004). Because elF1 stabilizes elF1A binding and the eukaryotic-specific $\alpha$-helical element of elF3c interacts with elF1 (Figure 2D), this interaction network may stabilize elF1A binding, consistent with the low dissociation rate of eIF1A from h48S AUG open. Furthermore, the $\alpha$-helical element of elF3c bridges between its CTD in the elF3 core and its NTD on the 40S intersubunit interface, where it blocks 60S subunit joining (Figure 2-figure supplement $1 \mathrm{C}$ ). In h48S AUG closed, the density of elF3c $\alpha$-helical element is not observed, indicating a structural rearrangement of the elF3 subunits upon start codon recognition, which might affect elF1A binding indirectly through release of elF1-elF3c contacts. These unique features of mammalian elF3 may explain its specific role in modulating the mammalian $48 \mathrm{~S} \mathrm{IC} \mathrm{conformation} \mathrm{compared} \mathrm{to} \mathrm{y48S} \mathrm{IC.}$

In summary, the principle conformational rearrangements of the 48S PIC induced by start codon recognition, e.g., $40 \mathrm{~S}$ subunit closure, movement of Met-tRNA ${ }_{i}^{M e t}$ and the displacement of elF1 and elF2 $\beta$ from the decoding site, appear to be conserved between lower and higher eukaryotes; however, their timing and regulation are notably different. Upon codon recognition, elF1A binding is destabilized in human, but stabilized in yeast system. elF3 is essential for start codon recognition in the mammalian system, but not in yeast. elF5 and GTP hydrolysis by elF2 are not necessary for codon recognition in mammalian system, consistent with the notion that elF5 is not essential for reconstitution of h48S IC. In mammals, start codon recognition relieves the elF1-gated inhibition of GTP hydrolysis by elF2 in the presence of elF5, which is necessary to remove elF2 from the complex (Majumdar and Maitra, 2005; Unbehaun et al., 2004). In contrast, in yeast elF5 plays a key role not only in 
promoting GTP hydrolysis and elF2 dissociation, but also in triggering Pi release from elF2 upon start codon recognition (Algire et al., 2005; Jennings and Pavitt, 2010; Nanda et al., 2013; Saini et al., 2014). In human system, elF1A binding is stabilized by elF5B after codon recognition, whereas in yeast elF5B binding appears to have no further effect on elF1A (Acker et al., 2009). The functional consequences of these differences in timing and regulation of start codon selection are not known, but may be related to the essential role of elF3 in translation regulation in mammals (Gomes-Duarte et al., 2018; Lee et al., 2015; Lee et al., 2016; Zeman et al., 2019), linked to complex networks of interactions with auxiliary regulators that play important roles in health and disease in mammalian cells. 


\section{Materials and Methods}

\section{Biochemical methods}

Model mRNAs. The model mRNA (GGG CAA CAA CAA CAA GCU AGC CACAA CAA CAA CAA CAA CAA CAA CAA GUC GAC CAA CAA CAA CAA CAA CAA CAA CAA CAA CUC GAG CAA CAA CAA CAA CAA CAA CAA CAA CAA GGA UCC AAA ACA GAC CACC AUG/AUC/CUC GUA CGU UUC AAG GCU UGA GCC CUC GUC ACU GCC CUG UGG GGC AAG GUG ACU CUG GAA GAA GUU GGU GGU GAG GCC CUG GGC AGG CUG CAG AGU GUG AGG GAA GGU CUG GUU GUC UAC CAA) is constructed using pUC19 and amplified with forward (5'ATCTAGAATTCTAATACGACTCACTATAGG-3') and reverse (5'-TTGGTAGACAACCAGACCTTCC3') primers. The PCR product was transcribed in vitro using T7 RNA-polymerase and purified by a Hitrap Q HP column (GE Healthcare) with ethanol precipitation.

Met-tRNA ${ }_{i}{ }^{\text {Met }}$ preparations. Full-length human $\operatorname{tRNA}_{i}{ }^{\text {Met }}$ sequence is inserted into the pIDTSMART-AMP plasmid (Integrated DNA Technologies) and amplified with forward (5'TAATACGACTCACTATAAGCAGAGTGG-3') and reverse (5'-TGGTAGCAGAGGATGGTTTCGATC$\left.3^{\prime}\right)$ primers. $\mathrm{tRNA}_{\mathrm{i}}{ }^{\mathrm{Met}}$ is prepared by in-vitro transcription using T7 RNA-polymerase and purified as described above for mRNA. tRNA was aminoacylated in the presence of S100 extract from E. coli (in-house made (Milon et al., 2007)) with $\left[{ }^{3} \mathrm{H}\right]$-methionine. $\left[{ }^{3} \mathrm{H}\right]$ MettRNA $A_{i}{ }^{M e t}$ is purified by reverse-phase chromatography (Milon et al., 2007).

Purification of recombinantly-expressed elFs (elF1, elF1A, elF4A, elF4B, and elF5). Sequences encoding full-length human eIF1, eIF1A, elF4A, elF4B, and elF5 were cloned into the pET24a vector without a tag or into ptxB1 vector (eIF5) with a cleavable intein-linked Histag. elF1, elF1A and elF5 were expressed in E. coli BL21 codon-plus RILP (Agilent Technologies); elF4A and elF4B were expressed in E. coli Rosetta strain. Recombinant proteins were purified on a HiTrap SP HP column (GE Healthcare) and subsequently on a HiTrap Q HP column (GE Healthcare) using a 50-1000 mM KCl gradient in buffer (20 mM Tris- $\mathrm{HCl}, \mathrm{pH} 7.5$, $0.1 \mathrm{mM}$ EDTA, 5\% glycerol, $2 \mathrm{mM}$ DTT) at $4{ }^{\circ} \mathrm{C}$. For elF4A, an additional HiTrap Blue column (GE Healthcare) was introduced after the HiTrap Q HP column. For elF5, an additional Protino Ni-IDA column (Macherey-Nagel) was applied before the Hitrap SP HP column and the His-tag was cleaved with $50 \mathrm{mM}$ DTT in buffer (20 mM Tris- $\mathrm{HCl}$, pH 7.5, $0.1 \mathrm{mM}$ EDTA, 5\% glycerol, 
$100 \mathrm{mM} \mathrm{KCl}$ ). Recombinant proteins were identified by SDS-PAGE (12\%) and confirmed by mass spectrometry.

elF1A derivatives (N4C, S74C, T120C) were prepared in the same way as described above. Purified elF1A derivatives were labeled at $4{ }^{\circ} \mathrm{C}$ with 4 -fold excess of Alexa Fluor ${ }^{\mathrm{TM}} 555 \mathrm{C} 2$ maleimide (Alexa555, Thermo Scientific) following the manufacturer's manual. To remove the excess free dye, elF1A preparations were re-purified on a HiTrap SP HP column and the fractions containing labeled elF1A visualized on a $15 \%$ SDS-PAGE using a fluorescence scanner (Typhoon ${ }^{\mathrm{TM}}$ FLA7000 (GE Healthcare)).

Purification of native human factors (elF2, elF3, elF5B, eEF1A and eEF2), 40S and 60S subunits. Native human initiation factors and ribosomes were prepared from HeLa cytoplasmic lysate as described (Pisarev et al., 2007). In brief, the polysomes were isolated from the HeLa cytoplasmic lysate pelleting through a $2 \mathrm{M}$ sucrose cushion in buffer $(20 \mathrm{mM}$ Tris- $\mathrm{HCl}$, pH 7.5, $20 \mathrm{mM} \mathrm{KCl}, 4 \mathrm{mM} \mathrm{MgCl}$, 2 mM DTT) by 18-hour centrifugation with a Ti 50.2 rotor at 45000 r.p.m. at $4{ }^{\circ} \mathrm{C}$. After resuspension of the polysomes, ribosome-bound factors were washed-off by increasing $\mathrm{KCl}$ concentration to $0.5 \mathrm{M}$. Factor-free polysomes were pelleted by centrifugation in a Ti 50.2 rotor at 45000 r.p.m at $4{ }^{\circ} \mathrm{C}$ for $4.5 \mathrm{hr}$. Mixtures of factors were then precipitated by increasing ammonium sulfate concentration to $40 \%$ and after pelleting to $50 \%$. The $40 \%$ ammonium sulfate fraction is used for elF3 purification and the $40-50 \%$ ammonium sulfate fraction is a source for elF2 and elF5B. After ammonium sulfate precipitation, proteins were resuspended and factors were purified on a HiTrap SP HP column using a 100-1000 mM KCl gradient in buffer (20 mM Tris- $\mathrm{HCl}, \mathrm{pH} 7.5,0.1 \mathrm{mM}$ EDTA $\mathrm{pH} 8,5 \%$ glycerol, $2 \mathrm{mM}$ DTT), followed by an additional purification step on a MonoQ 5/50 GL column (GE Healthcare) at $4{ }^{\circ} \mathrm{C}$. The elongation factors, eEF1A and eEF2, were purified in a similar way from $30-70 \%$ ammonium sulfate fraction as described previously (Pestova and Hellen, 2005). The purity of native initiation factors was confirmed by gradient SDS-PAGE gels and mass spectrometry. Notably, purified native human elF3 contains significant amount of elF4G, which prompted us to supply additional elF4A and elF4B in the reconstituted complexes. This increased the initiation efficiency only slightly; nevertheless, we kept the factors in the reaction mixture for better comparison with other experiments.

For human ribosome purification, factor-free polysome fraction was resuspended in buffer (20 mM Tris- $\mathrm{HCl}, \mathrm{pH} 7.5,4 \mathrm{mM} \mathrm{MgCl} 2,50 \mathrm{mM} \mathrm{KCl}, 2 \mathrm{mM} \mathrm{DTT}$ ) at $4{ }^{\circ} \mathrm{C}$ and treated with $1 \mathrm{mM}$ 
puromycin to release nascent peptides at $37^{\circ} \mathrm{C}$ for $10 \mathrm{~min}$. $80 \mathrm{~S}$ ribosomes were split into $40 \mathrm{~S}$ and $60 \mathrm{~S}$ subunits by increasing $\mathrm{KCl}$ concentration to $0.5 \mathrm{M}$ at $4{ }^{\circ} \mathrm{C}$. Mixtures of $40 \mathrm{~S}$ and $60 \mathrm{~S}$ subunits were applied to an ice-cold $10-30 \%$ sucrose gradient and separated by overnight centrifugation using an SW32 Ti rotor at $4{ }^{\circ} \mathrm{C}$. Fractions containing $40 \mathrm{~S}$ and $60 \mathrm{~S}$ subunits were identified by $260 \mathrm{~nm}$ absorbance and confirmed by mass spectrometry.

48S IC preparation. Human 48S complexes were assembled in buffer (20 mM Hepes, pH 7.5, $95 \mathrm{mM}$ KOAc, $3.75 \mathrm{mM} \mathrm{Mg}(\mathrm{OAc})_{2}, 1 \mathrm{mM}$ ATP, $0.5 \mathrm{mM}$ GTP, $0.25 \mathrm{mM}$ spermidine, $2 \mathrm{mM}$ DTT, $0.4 \mathrm{U} / \mu \mathrm{L}$ RiboLock RNase inhibitor) with $0.36 \mu \mathrm{M}$ 40S subunits, $1.1 \mu \mathrm{M}$ elF1, elF1A, elF4A, and mRNA each, $0.54 \mu \mathrm{M}$ elF3 and elF4B each, $0.72 \mu \mathrm{M}$ elF2, and $0.8 \mu \mathrm{M}$ Met-tRNA ${ }^{\text {Met }}$ at $37^{\circ} \mathrm{C}$ for $10 \mathrm{~min}$. To assemble 80S IC, additional $3.6 \mu \mathrm{M}$ elF5, $1.1 \mu \mathrm{M}$ elF5B and $0.72 \mu \mathrm{M} 60 \mathrm{~S}$ subunits were added after $48 \mathrm{~S} \mathrm{IC} \mathrm{formation} \mathrm{at} 37^{\circ} \mathrm{C}$ for $10 \mathrm{~min}$.

Toe-printing assay. In the toe-printing assay, 48S IC and 80S IC were assembled as described above, except for the mRNA concentrations, which was $0.2 \mu \mathrm{M}$. Toe-printing primer (Atto647N-GACCTTCCCTCACACTCTG) $(0.05 \mu \mathrm{M})$ was added into the $48 \mathrm{~S} \mathrm{IC}$ and $80 \mathrm{~S} \mathrm{IC}$ mixtures in reverse-transcription buffer $(0.5 \mathrm{mM}$ dNTPs, $8 \mathrm{mM} \mathrm{MgCl} 2,0.15 \mathrm{U} / \mu \mathrm{L}$ SuperScript III reverse transcriptase (Invitrogen)) and the reaction was incubated for $45 \mathrm{~min}$ at $37^{\circ} \mathrm{C}$. The cDNA products were extracted with phenol:chloroform:isoamyl alcohol and analyzed on an 8\% urea-PAGE using fluorescence scanner (Typhoon ${ }^{\text {TM }}$ FLA7000, GE Healthcare).

Spectrofluorometer assays. To monitor fluorescence changes of elF1A upon $48 \mathrm{~S}$ IC formation, $0.06 \mu \mathrm{M}$ labeled elF1A variants were used in each measurement and components to assemble the 48S IC were added step-wise into the reaction mixture at the same concentration and condition as described above. 3.6 $\mu \mathrm{M}$ unlabeled elF1A was added to chase the labeled eIF1A variants from 48S IC. After adding each component, the fluorescence intensity and anisotropy were measured at $25{ }^{\circ} \mathrm{C}$ with excitation at $555 \mathrm{~nm}$ and emission at $568 \mathrm{~nm} .5$ technical replicates were taken for each measurement. For determinations of $\mathrm{K}_{\mathrm{d}}$ and $K_{i}$ of elF1A to the 40 S subunit, 5 nM elF1A(C4-Alx555) or $5 \mathrm{nM} 40$ S-elF1A(C4-Alx555) complex were mixed with increasing concentrations of the $40 \mathrm{~S}$ subunit or unlabeled elF1A, respectively. The anisotropy was measured in the same condition as described above. 
Chase experiments. $43 \mathrm{~S}$ and $48 \mathrm{~S}$ complexes were assembled as described above using 0.06 $\mu \mathrm{M}$ Alexa555-labeled elF1A variants. Additionally, $0.05 \%$ bovine serum albumin was included in the buffer to prevent non-specific binding of labeled elF1A to the cuvette walls. In the conditions of $48 \mathrm{~S}+\mathrm{elF} 5$ or +elF5B, additional $3.6 \mu \mathrm{M}$ elF5 or $1.1 \mu \mathrm{M}$ elF5B were included. The $48 \mathrm{~S}$ complexes were rapidly mixed with $7.2 \mu \mathrm{M}$ of unlabeled WT elF1A at $25^{\circ} \mathrm{C}$ in the stoppedflow apparatus (SX-20MV (Applied Photophysics)). For dissociation of elF1A from 80S IC, the $48 \mathrm{~S}+\mathrm{elF} 5+\mathrm{elF} 5 \mathrm{~B}$ complex was rapidly mixed with $5.4 \mu \mathrm{M} 60 \mathrm{~S}$ subunit at $25^{\circ} \mathrm{C}$ in a stoppedflow apparatus. The fluorescence intensity was recorded with 4000 time points in logarithmic spacing using $535 \mathrm{~nm}$ excitation and a $570 \mathrm{~nm}$ emission filter. 5 technical replicates of time courses were collected for each experiment, averaged, and analyzed by single or double exponential fitting by GraphPad Prism.

\section{Cryo-EM methods}

GraFix and Cryo-EM grid preparation. Complex preparation for cryo-EM was carried out in buffer (20 mM Hepes, pH 7.5, 95 mM KOAc, 3.75 mM Mg(OAc)2, 1 mM ATP, 0.5 mM GTP ) at $4^{\circ} \mathrm{C}$. The complexes were stabilized for cryo-EM grid preparation by the GraFix approach (Kastner et al., 2008) with some modifications. Specifically, complexes were stabilized before gradient centrifugation by 30 min incubation with $2 \mathrm{mM}$ of a mild crosslinking reagent bis(sulfosuccinimidyl)suberate (BS3, Sigma Aldrich) at room temperature. Subsequently, complexes were crosslinked upon ultra-centrifugation on a linear $10-40 \%$ sucrose gradient (total volume $4.4 \mathrm{ml}$ run for $16 \mathrm{~h}$ at $138,000 \times \mathrm{g}$ ) combined with linear gradients of $0-0.1 \%$ glutaraldehyde (EM grade 25\%, Science Services GmbH, Munich, Germany) and 0-1.0 mM pmaleimidophenyl isocyanate (PMPI, ThermoFischer Scientific). PMPI was introduced because of its heterobifunctional activity in crosslinking RNA and proteins. The gradient was fractionated into $200 \mu \mathrm{l}$ fractions and the crosslinking reaction was quenched using $100 \mathrm{mM}$ aspartate (Sigma Aldrich) at $\mathrm{pH}$ 7.5. Sucrose was removed using Zeba Spin columns (ThermoFischer Scientific), which were pre-incubated with $0.1 \mathrm{ml} / \mathrm{mg}$ gelatin (Sigma Aldrich) and then washed with buffer to improve sample recovery. Cryo-EM grids were prepared by floating home-made continuous carbon on $40 \mu \mathrm{l}$ sample in the wells of teflon block (custommade). The sample-covered carbon was then adsorbed to an EM grid (Quantifoil R3.5/1, Jena Bioscience) and blotted for $9 \mathrm{~s}$ using a Vitrobot Mark IV (ThermoFisher, Eindhoven) operated at $4^{\circ} \mathrm{C}$ and $100 \%$ humidity. 
Data acquisition. Cryo-EM data acquisition was performed using a Falcon III direct electron detector (ThermoFisher, Eindhoven) on a Titan Krios G1 microscope with 300 kV acceleration voltage equipped with an XFEG electron source and a $\mathrm{C}_{\mathrm{s}}$-corrector (CEOS, Heidelberg) aligned with the CETCORPLUS 4.6.9 (CEOS, Heidelberg) software package. The total dataset of 15,544 cryo-EM movies (4096 x 4096 pixels) with 20 frames each was acquired using EPU 1.11 (ThermoFisher Eindhoven) in integration mode and $1 \mathrm{~s}$ exposure time at total electron dose of 48 electrons per $\AA^{2}$ and a defocus range of 1.5-4.0 $\mu \mathrm{m}$.

Data processing. Beam-induced motion correction with dose-weighting was performed with MotionCor2 (Zheng et al., 2017) using $5 \times 5$ patches. CTF parameters of the motion-corrected micrographs were determined by Gctf (Zhang, 2016) and 990,486 particles particle were selected with Gautomatch (K. Zhang, MRC-LMB, Cambridge). All subsequent image processing was performed using RELION 3.0. The selected particles were extracted at $2.32 \AA /$ pixel and sorted in a hierarchical manner using RELION 3.0 (Figure 1 - figure supplement 3A). 2D classification reduced the total number of particles to 821,651 , which were then refined and sorted for 405 head conformation by focused 3D classification with signal subtraction without alignment using a mask for the $40 \mathrm{~S}$ head (top panel in Figure 1 - figure supplement 3B) revealing two major populations with an open and a closed conformation of the 405 head, respectively. Subsequently, each of the particle populations was sorted separately focusing on the region of the decoding center and ternary complex area by 3D classification with signal subtraction (bottom panel in Figure 1 - figure supplement 3B). The resulting particle populations were each re-extracted at the final pixel size of $1.16 \mathrm{~A} / \mathrm{pix}$, their CTF parameters were locally determined using CTF refinement and the particles were refined to highresolution following the gold-standard procedure using soft solvent masks (Figure 1 - figure supplement 3 C). Global amplitude sharpening of the two final maps was performed using Phenix 1.16-3549 (Liebschner et al., 2019). To estimate the occupancy with elF3, the final particle populations of the h48S open and closed state were each refined against the isolated 405 subunit to avoid any reference bias for elF3 and then sorted into ten classes by focused classification with signal subtraction using a mask on the core of elF3. In each case, all classes showed clear density for the elF3 core indicating a high occupancy above $80 \%$ in both states. 
Atomic model refinement. Initial models were obtained by rigid-body fitting individual chains of the structure from the human $48 \mathrm{~S}$ complex in the scanning state (PDB 6ZMW, (Brito Querido et al., 2020)) into each of the two final cryo-EM maps using ChimeraX 1.2 (Pettersen et al., 2021), and subsequent manual adjustments and refinement in Coot 0.9.3 (Brown et al., 2015). Local atomic model refinement was done using low pass filtered to corresponding local resolutions of the maps (Figure 1 - figure supplement $3 E$ ) in Coot. For the elF3 core atomic model refinement was done at $6 \AA$, and at $12 \AA$ for peripheral subunits, elF $2 \gamma$ was fitted as rigid-body and refined with all-atom restraints at $12 \AA$, the $40 S$ subunit, and the decoding center area of ternary complex was refined at the respective final resolutions, i.e. $3.7 \AA$ and 4.7 $\AA$, and elF1A was refined at $6 \AA$ resolution. The mRNA in both states was first modeled manually in Coot and then refined in ISOLDE 1.1.0 (Croll, 2018). Nucleotide bases for the mRNA in the open state were omitted, due to the weak density for the mRNA in this state. The resulting initial models were then automatically refined using phenix.real_space_refine (Liebschner et al., 2019) with secondary structure restraints for 10 macrocycles 500 iterations each.

\section{Crosslinking-mass spectrometry}

The reconstituted 48S AUG IC was treated with either 2 mM BS3 (ThermoFisherScientific) or $2 \mathrm{mM}$ LC-SDA (ThermoFisherScientific) for $1 \mathrm{~h}$ at RT. For LC-SDA crosslinking, the complex was dialyzed against buffer (20 mM Hepes, pH 7.5, 95 mM KOAc, 3.75 mM Mg(OAc)2, 1 mM ATP, $0.5 \mathrm{mM}$ GTP) via a membrane filter (MF Membrane Filters, $0.025 \mu \mathrm{m}$ VSWP, Merck) prior to crosslinking. LC-SDA crosslinked samples were irradiated with UV light ( $365 \mathrm{~nm}$ ) for $5 \mathrm{~min}$ at $4^{\circ} \mathrm{C}$. Crosslinking reaction with $\mathrm{Bs} 3$ or LC-SDA were quenched with $50 \mathrm{mM}$ Tris- $\mathrm{HCl} \mathrm{pH} 7.5$ for 15 min. (Crosslinked) proteins were reduced with $10 \mathrm{mM}$ dithiothreitol and subsequently alkylated with $40 \mathrm{mM}$ iodoacetamide under standard conditions. Proteins were digested with the endoproteinase trypsin in an enzyme-to-protein ratio of 1:50 in the presence of $1 \mathrm{M}$ urea at $37^{\circ} \mathrm{C}$ overnight. The reaction was terminated with $0.5 \%$ trifluoroacetic acid (TFA) $(\mathrm{v} / \mathrm{v})$, the peptide mixtures were desalted on MicroSpin Columns (Harvard Apparatus) following manufacturer's instructions and vacuum-dried in a SpeedVac. Peptides were dissolved in 50 $\mu \mathrm{L} 30 \%$ acetonitrile $(\mathrm{v} / \mathrm{v})$ in water $/ 0.1 \%(\mathrm{v} / \mathrm{v})$ trifluoroacetic acid (TFA) and crosslinked peptides were enriched by peptide size exclusion chromatography (SuperdexPeptide 3.2/300 column, GE Healthcare) with a flow rate of $50 \mu \mathrm{l} / \mathrm{min}$ in $30 \%(\mathrm{v} / \mathrm{v})$ acetonitrile with water 
and $0.1 \%$ (v/v) TFA (Gomkale et al., 2021; Singh et al., 2020). Fractions of $50 \mu \mathrm{L}$ were collected and early eluting fractions that contained crosslinked peptides were subjected to liquid chromatography-coupled -mass spectrometry (LC-MS)) analyses.

LC-MS analysis was performed as described (Gomkale et al., 2021; Singh et al., 2020). In brief, for each crosslinker crosslinked peptides were determined as technical duplicates using an Orbitrap QExactive HF Mass Spectrometer coupled to a Dionex UltiMate 3000 UHPLC system (both Thermo Fisher Scientific) that was equipped with an in house-packed C18 column (ReproSil-Pur $120 \mathrm{C} 18-\mathrm{AQ}, 1.9 \mu \mathrm{m}$ pore size, $75 \mu \mathrm{m}$ inner diameter, $30 \mathrm{~cm}$ length, $\mathrm{Dr}$. Maisch $\mathrm{GmbH}$ ). First mass spectrometer (MS1) full scans were acquired with a resolution of 120,000 , an injection time (IT) of $60 \mathrm{~ms}$ and an automatic gain control (AGC) target of $1 \times 10^{6}$. Dynamic exclusion (DE) was set to $30 \mathrm{~s}$ and only charge states between 3 and 8 were considered for fragmentation. MS2 spectra were acquired of the 30 most abundant precursor ions; the resolution was set to 30,000 ; the IT to $128 \mathrm{~ms}$ and the $\mathrm{AGC}$ target to $1 \times 10^{5}$. Fragmentation was enforced by higher-energy collisional dissociation (HCD) at 30\% NCE.

Raw files were analyzed by pLink 2.3.9 (Chen et al., 2019) for the identification of crosslinked peptides against the full set of $H$. sapiens initiation factors and ribosomal proteins of the $40 S$ subunit retrieved from the UniProt database (Bateman et al., 2021). False discovery rate (FDR) was set below $5 \%$ on spectrum level; crosslinked peptide spectrum matches (CSMs) were not evaluated manually. For each crosslinker identified crosslinks were first filtered to include only unambiguous crosslinks with $\geq 2$ hits and a maximum - $\log _{10}$ (E-value) $\geq 3$ (from pLink 2.3.9) and then mapped onto our structural models of the open and closed h48S IC states using the software Xlink Analyzer 1.1.4 (Kosinski et al., 2015) and Chimera 1.15 (Pettersen et al., 2004). In total, we identified 748 and 714 unique crosslinks that could be mapped onto the models of the open and closed states, respectively. For the open state, 297 and 451 crosslinks could be mapped with LC-SDA and BS3, respectively, and for the closed state 280 crosslinks with LC-SDA and 437 with BS3. 


\section{Author contributions}

S.-H.Y., N.F., A.G, A.C., H.S., S.A., and M.V.R. conceived the experiments; S.-H.Y. and A.G. performed biochemical and kinetic experiments with the input of S.A.; S.-H.Y. analyzed the kinetic data; V.P., J.E.S., and N.F. performed cryo-EM experiments; A.L. and H.U. performed mass spectrometry analysis. S.-H.Y., V.P., N.F., S.A., and M.V.R. wrote the manuscript with input from all authors. All authors discussed the data analysis, critically reviewed the manuscript and approved the final version.

\section{Acknowledgements}

We thank Prof. Dr. Tatyana Pestova for introducing us into the mammalian initiation system, generous guidance on biochemical material preparations and insightful comments on the manuscript; Thomas Conrad and Hossein Kohansal for HeLa cell extract; Olaf Geintzer, Vanessa Herold, Tessa Hübner, Franziska Hummel, Sandra Kappler, Christina Kothe, Anna Pfeifer, Lena Preiser, Theresia Steiger, and Michael Zimmermann for expert technical assistance. The work was funded by the Max Planck Society and the grants of the Deutsche Forschungsgemeinschaft (SFB860 to S.A., M.V.R. and H.S.; Leibniz Prize to M.V.R.). A.G. acknowledges the support of Boehringer Ingelheim Fonds.

\section{Declaration of interests}

The authors declare no competing interests.

\section{Data availability}

Final cryo-EM maps/atomic coordinates have been deposited in the EM database/Protein Data Bank with accession codes EMD-XXXX/PDB YYYY for the h48S AUG open state and EMDXXXX/PDB YYYY for the h48S AUG closed state, respectively. Cryo-EM raw data have been deposited in the EMPIAR database as entry EMPIAR-ZZZZ. 


\section{References}

Acker, M.G., Shin, B.S., Dever, T.E., and Lorsch, J.R. (2006). Interaction between eukaryotic initiation factors $1 A$ and $5 B$ is required for efficient ribosomal subunit joining. J Biol Chem 281, 8469-8475.

Acker, M.G., Shin, B.S., Nanda, J.S., Saini, A.K., Dever, T.E., and Lorsch, J.R. (2009). Kinetic analysis of late steps of eukaryotic translation initiation. J Mol Biol 385, 491-506.

Algire, M.A., Maag, D., and Lorsch, J.R. (2005). Pi release from elF2, not GTP hydrolysis, is the step controlled by start-site selection during eukaryotic translation initiation. Mol Cell 20, 251-262.

Algire, M.A., Maag, D., Savio, P., Acker, M.G., Tarun, S.Z., Jr., Sachs, A.B., Asano, K., Nielsen, K.H., Olsen, D.S., Phan, L., et al. (2002). Development and characterization of a reconstituted yeast translation initiation system. RNA 8, 382-397.

Bateman, A., Martin, M.J., Orchard, S., Magrane, M., Agivetova, R., Ahmad, S., Alpi, E., Bowler-Barnett, E.H., Britto, R., Bursteinas, B., et al. (2021). UniProt: the universal protein knowledgebase in 2021. Nucleic Acids Research 49, D480-D489.

Battiste, J.L., Pestova, T.V., Hellen, C.U., and Wagner, G. (2000). The elF1A solution structure reveals a large RNA-binding surface important for scanning function. Mol Cell 5, 109-119.

Brito Querido, J., Sokabe, M., Kraatz, S., Gordiyenko, Y., Skehel, J.M., Fraser, C.S., and Ramakrishnan, V. (2020). Structure of a human $48 S$ translational initiation complex. Science 369, 1220-1227.

Brown, A., Long, F., Nicholls, R.A., Toots, J., Emsley, P., and Murshudov, G. (2015). Tools for macromolecular model building and refinement into electron cryo-microscopy reconstructions. Acta Crystallogr D Biol Crystallogr 71, 136-153.

Chen, Z.L., Meng, J.M., Cao, Y., Yin, J.L., Fang, R.Q., Fan, S.B., Liu, C., Zeng, W.F., Ding, Y.H., Tan, D., et al. (2019). A high-speed search engine pLink 2 with systematic evaluation for proteome-scale identification of cross-linked peptides. Nat Commun 10.

Cheung, Y.N., Maag, D., Mitchell, S.F., Fekete, C.A., Algire, M.A., Takacs, J.E., Shirokikh, N., Pestova, T., Lorsch, J.R., and Hinnebusch, A.G. (2007). Dissociation of elF1 from the $40 \mathrm{~S}$ ribosomal subunit is a key step in start codon selection in vivo. Genes Dev 21, 1217-1230.

Costa-Mattioli, M., and Walter, P. (2020). The integrated stress response: From mechanism to disease. Science 368.

Croll, T.I. (2018). ISOLDE: a physically realistic environment for model building into lowresolution electron-density maps. Acta Crystallogr D Struct Biol 74, 519-530.

des Georges, A., Dhote, V., Kuhn, L., Hellen, C.U., Pestova, T.V., Frank, J., and Hashem, Y. (2015). Structure of mammalian elF3 in the context of the 43S preinitiation complex. Nature 525, 491-495. 
Eliseev, B., Yeramala, L., Leitner, A., Karuppasamy, M., Raimondeau, E., Huard, K., Alkalaeva, E., Aebersold, R., and Schaffitzel, C. (2018). Structure of a human cap-dependent $48 \mathrm{~S}$ translation pre-initiation complex. Nucleic Acids Res 46, 2678-2689.

Fekete, C.A., Mitchell, S.F., Cherkasova, V.A., Applefield, D., Algire, M.A., Maag, D., Saini, A.K., Lorsch, J.R., and Hinnebusch, A.G. (2007). N- and C-terminal residues of elF1A have opposing effects on the fidelity of start codon selection. EMBO J 26, 1602-1614.

Fraser, C.S., Berry, K.E., Hershey, J.W., and Doudna, J.A. (2007). elF3j is located in the decoding center of the human 40 S ribosomal subunit. Mol Cell 26, 811-819.

Fringer, J.M., Acker, M.G., Fekete, C.A., Lorsch, J.R., and Dever, T.E. (2007). Coupled release of eukaryotic translation initiation factors $5 B$ and $1 A$ from $80 S$ ribosomes following subunit joining. Mol Cell Biol 27, 2384-2397.

Gomes-Duarte, A., Lacerda, R., Menezes, J., and Romao, L. (2018). elF3: a factor for human health and disease. RNA Biol 15, 26-34.

Gomkale, R., Linden, A., Neumann, P., Schendzielorz, A.B., Stoldt, S., Dybkov, O., Kilisch, M., Schulz, C., Cruz-Zaragoza, L.D., Schwappach, B., et al. (2021). Mapping protein interactions in the active TOM-TIM23 supercomplex. Nat Commun 12.

Hashem, Y., des Georges, A., Dhote, V., Langlois, R., Liao, H.Y., Grassucci, R.A., Hellen, C.U., Pestova, T.V., and Frank, J. (2013a). Structure of the mammalian ribosomal 435 preinitiation complex bound to the scanning factor DHX29. Cell 153, 1108-1119.

Hashem, Y., des Georges, A., Dhote, V., Langlois, R., Liao, H.Y., Grassucci, R.A., Pestova, T.V., Hellen, C.U., and Frank, J. (2013b). Hepatitis-C-virus-like internal ribosome entry sites displace elF3 to gain access to the 40 S subunit. Nature $503,539-543$.

Hussain, T., Llacer, J.L., Fernandez, I.S., Munoz, A., Martin-Marcos, P., Savva, C.G., Lorsch, J.R., Hinnebusch, A.G., and Ramakrishnan, V. (2014). Structural changes enable start codon recognition by the eukaryotic translation initiation complex. Cell 159, 597-607.

Jennings, M.D., and Pavitt, G.D. (2010). elF5 has GDI activity necessary for translational control by elF2 phosphorylation. Nature 465, 378-381.

Kapp, L.D., and Lorsch, J.R. (2004). GTP-dependent recognition of the methionine moiety on initiator tRNA by translation factor elF2. J Mol Biol 335, 923-936.

Kastner, B., Fischer, N., Golas, M.M., Sander, B., Dube, P., Boehringer, D., Hartmuth, K., Deckert, J., Hauer, F., Wolf, E., et al. (2008). GraFix: sample preparation for single-particle electron cryomicroscopy. Nat Methods 5, 53-55.

Kosinski, J., von Appen, A., Ori, A., Karius, K., Muller, C.W., and Beck, M. (2015). Xlink Analyzer: Software for analysis and visualization of cross-linking data in the context of threedimensional structures. Journal of Structural Biology 189, 177-183. 
Kumar, P., Hellen, C.U., and Pestova, T.V. (2016). Toward the mechanism of elF4F-mediated ribosomal attachment to mammalian capped mRNAs. Genes Dev 30, 1573-1588.

Lee, A.S., Kranzusch, P.J., and Cate, J.H. (2015). elF3 targets cell-proliferation messenger RNAs for translational activation or repression. Nature 522, 111-114.

Lee, A.S., Kranzusch, P.J., Doudna, J.A., and Cate, J.H. (2016). elF3d is an mRNA cap-binding protein that is required for specialized translation initiation. Nature 536, 96-99.

Lee, J.H., Pestova, T.V., Shin, B.S., Cao, C., Choi, S.K., and Dever, T.E. (2002). Initiation factor elF5B catalyzes second GTP-dependent step in eukaryotic translation initiation. Proc Natl Acad Sci U S A 99, 16689-16694.

Liebschner, D., Afonine, P.V., Baker, M.L., Bunkoczi, G., Chen, V.B., Croll, T.I., Hintze, B., Hung, L.W., Jain, S., McCoy, A.J., et al. (2019). Macromolecular structure determination using X-rays, neutrons and electrons: recent developments in Phenix. Acta Crystallogr D Struct Biol 75, 861-877.

Lin, K.Y., Nag, N., Pestova, T.V., and Marintchev, A. (2018). Human elF5 and elF1A Compete for Binding to elF5B. Biochemistry 57, 5910-5920.

Llacer, J.L., Hussain, T., Marler, L., Aitken, C.E., Thakur, A., Lorsch, J.R., Hinnebusch, A.G., and Ramakrishnan, V. (2015). Conformational Differences between Open and Closed States of the Eukaryotic Translation Initiation Complex. Mol Cell 59, 399-412.

Llacer, J.L., Hussain, T., Saini, A.K., Nanda, J.S., Kaur, S., Gordiyenko, Y., Kumar, R., Hinnebusch, A.G., Lorsch, J.R., and Ramakrishnan, V. (2018). Translational initiation factor eIF5 replaces eIF1 on the 40S ribosomal subunit to promote start-codon recognition. Elife 7.

Lomakin, I.B., and Steitz, T.A. (2013). The initiation of mammalian protein synthesis and mRNA scanning mechanism. Nature 500, 307-311.

Luna, R.E., Arthanari, H., Hiraishi, H., Akabayov, B., Tang, L., Cox, C., Markus, M.A., Luna, L.E., Ikeda, Y., Watanabe, R., et al. (2013). The interaction between eukaryotic initiation factor $1 \mathrm{~A}$ and elF5 retains elF1 within scanning preinitiation complexes. Biochemistry 52, 9510-9518.

Luna, R.E., Arthanari, H., Hiraishi, H., Nanda, J., Martin-Marcos, P., Markus, M.A., Akabayov, B., Milbradt, A.G., Luna, L.E., Seo, H.C., et al. (2012). The C-terminal domain of eukaryotic initiation factor 5 promotes start codon recognition by its dynamic interplay with elF1 and elF2beta. Cell Rep 1, 689-702.

Maag, D., Algire, M.A., and Lorsch, J.R. (2006). Communication between eukaryotic translation initiation factors 5 and $1 \mathrm{~A}$ within the ribosomal pre-initiation complex plays a role in start site selection. J Mol Biol 356, 724-737.

Maag, D., Fekete, C.A., Gryczynski, Z., and Lorsch, J.R. (2005). A conformational change in the eukaryotic translation preinitiation complex and release of elF1 signal recognition of the start codon. Mol Cell 17, 265-275. 
Maag, D., and Lorsch, J.R. (2003). Communication between eukaryotic translation initiation factors 1 and $1 \mathrm{~A}$ on the yeast small ribosomal subunit. J Mol Biol 330, 917-924.

Majumdar, R., Bandyopadhyay, A., and Maitra, U. (2003). Mammalian translation initiation factor elF1 functions with elF1A and eIF3 in the formation of a stable $40 \mathrm{~S}$ preinitiation complex. J Biol Chem 278, 6580-6587.

Majumdar, R., and Maitra, U. (2005). Regulation of GTP hydrolysis prior to ribosomal AUG selection during eukaryotic translation initiation. EMBO J 24, 3737-3746.

Milon, P., Konevega, A.L., Peske, F., Fabbretti, A., Gualerzi, C.O., and Rodnina, M.V. (2007). Transient kinetics, fluorescence, and FRET in studies of initiation of translation in bacteria. Methods Enzymol 430, 1-30.

Moon, S.L., and Parker, R. (2018). EIF2B2 mutations in vanishing white matter disease hypersuppress translation and delay recovery during the integrated stress response. RNA 24, 841-852.

Nanda, J.S., Cheung, Y.N., Takacs, J.E., Martin-Marcos, P., Saini, A.K., Hinnebusch, A.G., and Lorsch, J.R. (2009). elF1 controls multiple steps in start codon recognition during eukaryotic translation initiation. J Mol Biol 394, 268-285.

Nanda, J.S., Saini, A.K., Munoz, A.M., Hinnebusch, A.G., and Lorsch, J.R. (2013). Coordinated movements of eukaryotic translation initiation factors elF1, elF1A, and elF5 trigger phosphate release from elF 2 in response to start codon recognition by the ribosomal preinitiation complex. J Biol Chem 288, 5316-5329.

Obayashi, E., Luna, R.E., Nagata, T., Martin-Marcos, P., Hiraishi, H., Singh, C.R., Erzberger, J.P., Zhang, F., Arthanari, H., Morris, J., et al. (2017). Molecular Landscape of the Ribosome Pre-initiation Complex during mRNA Scanning: Structural Role for elF3c and Its Control by elF5. Cell Rep 18, 2651-2663.

Passmore, L.A., Schmeing, T.M., Maag, D., Applefield, D.J., Acker, M.G., Algire, M.A., Lorsch, J.R., and Ramakrishnan, V. (2007). The eukaryotic translation initiation factors elF1 and elF1A induce an open conformation of the $40 \mathrm{~S}$ ribosome. Mol Cell 26, 41-50.

Pestova, T.V., Borukhov, S.I., and Hellen, C.U.T. (1998). Eukaryotic ribosomes require initiation factors 1 and $1 \mathrm{~A}$ to locate initiation codons. Nature 394, 854-859.

Pestova, T.V., and Hellen, C.U. (2005). Reconstitution of eukaryotic translation elongation in vitro following initiation by internal ribosomal entry. Methods 36, 261-269.

Pestova, T.V., and Kolupaeva, V.G. (2002). The roles of individual eukaryotic translation initiation factors in ribosomal scanning and initiation codon selection. Genes Dev 16, 29062922.

Pestova, T.V., Lomakin, I.B., Lee, J.H., Choi, S.K., Dever, T.E., and Hellen, C.U. (2000). The joining of ribosomal subunits in eukaryotes requires elF5B. Nature 403, 332-335. 
Pettersen, E.F., Goddard, T.D., Huang, C.C., Couch, G.S., Greenblatt, D.M., Meng, E.C., and Ferrin, T.E. (2004). UCSF chimera - A visualization system for exploratory research and analysis. J Comput Chem 25, 1605-1612.

Pettersen, E.F., Goddard, T.D., Huang, C.C., Meng, E.C., Couch, G.S., Croll, T.I., Morris, J.H., and Ferrin, T.E. (2021). UCSF ChimeraX: Structure visualization for researchers, educators, and developers. Protein Sci 30, 70-82.

Pisarev, A.V., Kolupaeva, V.G., Yusupov, M.M., Hellen, C.U., and Pestova, T.V. (2008). Ribosomal position and contacts of $m R N A$ in eukaryotic translation initiation complexes. EMBO J 27, 1609-1621.

Pisarev, A.V., Skabkin, M.A., Pisareva, V.P., Skabkina, O.V., Rakotondrafara, A.M., Hentze, M.W., Hellen, C.U., and Pestova, T.V. (2010). The role of ABCE1 in eukaryotic posttermination ribosomal recycling. Mol Cell 37, 196-210.

Pisarev, A.V., Unbehaun, A., Hellen, C.U., and Pestova, T.V. (2007). Assembly and analysis of eukaryotic translation initiation complexes. Methods Enzymol 430, 147-177.

Pisareva, V.P., Hellen, C.U., and Pestova, T.V. (2007). Kinetic analysis of the interaction of guanine nucleotides with eukaryotic translation initiation factor elF5B. Biochemistry 46, 2622-2629.

Pisareva, V.P., and Pisarev, A.V. (2014). elF5 and elF5B together stimulate 48 S initiation complex formation during ribosomal scanning. Nucleic Acids Res 42, 12052-12069.

Robichaud, N., Sonenberg, N., Ruggero, D., and Schneider, R.J. (2019). Translational Control in Cancer. Cold Spring Harb Perspect Biol 11.

Saini, A.K., Nanda, J.S., Martin-Marcos, P., Dong, J., Zhang, F., Bhardwaj, M., Lorsch, J.R., and Hinnebusch, A.G. (2014). Eukaryotic translation initiation factor elF5 promotes the accuracy of start codon recognition by regulating Pi release and conformational transitions of the preinitiation complex. Nucleic Acids Res 42, 9623-9640.

Schuller, A.P., and Green, R. (2017). The ABC(E1)s of Ribosome Recycling and Reinitiation. Mol Cell 66, 578-580.

Simonetti, A., Brito Querido, J., Myasnikov, A.G., Mancera-Martinez, E., Renaud, A., Kuhn, L., and Hashem, Y. (2016). elF3 Peripheral Subunits Rearrangement after mRNA Binding and Start-Codon Recognition. Mol Cell 63, 206-217.

Simonetti, A., Guca, E., Bochler, A., Kuhn, L., and Hashem, Y. (2020). Structural Insights into the Mammalian Late-Stage Initiation Complexes. Cell Rep 31, 107497.

Singh, K., Graf, B., Linden, A., Sautner, V., Urlaub, H., Tittmann, K., Stark, H., and Chari, A. (2020). Discovery of a Regulatory Subunit of the Yeast Fatty Acid Synthase. Cell 180, 1130-+. 
Sokabe, M., and Fraser, C.S. (2014). Human eukaryotic initiation factor 2 (elF2)-GTP-MettRNAi ternary complex and elF3 stabilize the $43 \mathrm{~S}$ preinitiation complex. J Biol Chem 289, 31827-31836.

Sokabe, M., and Fraser, C.S. (2017). A helicase-independent activity of elF4A in promoting mRNA recruitment to the human ribosome. Proc Natl Acad Sci U S A 114, 6304-6309.

Sokabe, M., Fraser, C.S., and Hershey, J.W. (2012). The human translation initiation multifactor complex promotes methionyl-tRNAi binding to the $40 \mathrm{~S}$ ribosomal subunit. Nucleic Acids Res 40, 905-913.

Unbehaun, A., Borukhov, S.I., Hellen, C.U., and Pestova, T.V. (2004). Release of initiation factors from $48 \mathrm{~S}$ complexes during ribosomal subunit joining and the link between establishment of codon-anticodon base-pairing and hydrolysis of elF2-bound GTP. Genes Dev 18, 3078-3093.

Valasek, L., Nielsen, K.H., Zhang, F., Fekete, C.A., and Hinnebusch, A.G. (2004). Interactions of eukaryotic translation initiation factor 3 (elF3) subunit NIP1/c with elF1 and elF5 promote preinitiation complex assembly and regulate start codon selection. Mol Cell Biol 24, 94379455.

Wang, J., Johnson, A.G., Lapointe, C.P., Choi, J., Prabhakar, A., Chen, D.H., Petrov, A.N., and Puglisi, J.D. (2019). elF5B gates the transition from translation initiation to elongation. Nature 573, 605-608.

Weisser, M., Voigts-Hoffmann, F., Rabl, J., Leibundgut, M., and Ban, N. (2013). The crystal structure of the eukaryotic $40 \mathrm{~S}$ ribosomal subunit in complex with elF1 and elF1A. Nat Struct Mol Biol 20, 1015-1017.

Zeman, J., Itoh, Y., Kukacka, Z., Rosulek, M., Kavan, D., Kouba, T., Jansen, M.E., Mohammad, M.P., Novak, P., and Valasek, L.S. (2019). Binding of elF3 in complex with elF5 and elF1 to the $40 \mathrm{~S}$ ribosomal subunit is accompanied by dramatic structural changes. Nucleic Acids Res $47,8282-8300$.

Zhang, K. (2016). Gctf: Real-time CTF determination and correction. Journal of Structural Biology 193, 1-12.

Zheng, S.Q., Palovcak, E., Armache, J.-P., Verba, K.A., Cheng, Y., and Agard, D.A. (2017). MotionCor2: anisotropic correction of beam-induced motion for improved cryo-electron microscopy. Nat Methods 14, 331-332. 